\title{
Rational Blanchfield forms, S-equivalence, and null LP-surgeries
}

\author{
Delphine Moussard
}

\begin{abstract}
Null Lagrangian-preserving surgeries are a generalization of the Garoufalidis and Rozansky null-moves, that these authors introduced to study the Kricker lift of the Kontsevich integral, in the setting of pairs $(M, K)$ composed of a rational homology sphere $M$ and a null-homologous knot $K$ in $M$. They are defined as replacements of null-homologous rational homology handlebodies of $M \backslash K$ by other such handlebodies with identical Lagrangian. A null Lagrangian-preserving surgery induces a canonical isomorphism between the Alexander $\mathbb{Q}\left[t^{ \pm 1}\right]$-modules of the involved pairs, which preserves the Blanchfield form. Conversely, we prove that a fixed isomorphism between Alexander $\mathbb{Q}\left[t^{ \pm 1}\right]$-modules which preserves the Blanchfield form can be realized, up to multiplication by a power of $t$, by a finite sequence of null Lagrangianpreserving surgeries. We also prove that such classes of isomorphisms can be realized by rational S-equivalences. In the case of integral homology spheres, we prove similar realization results for a fixed isomorphism between Alexander $\mathbb{Z}\left[t^{ \pm 1}\right]$-modules.
\end{abstract}

MSC: 57M25 57M27 57N10 57N65

Keywords: Alexander module; Blanchfield form; equivariant linking pairing; homology sphere; homology handlebody; Lagrangian-preserving surgery; Seifert matrix; S-equivalence; lift of the Kontsevich integral; null-move; Euler degree of the Kontsevich integral.

\section{Contents}

1 Introduction $\quad$

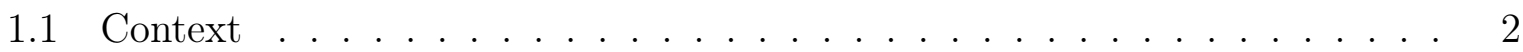

1.2 Alexander module and Blanchfield form . . . . . . . . . . . . . . . . . . . . . . . . . . . . . . .

1.3 Seifert matrices . . . . . . . . . . . . . . . . . . . . . . . 4 
1.4 S-equivalence . . . . . . . . . . . . . . . . . . 5

1.5 Lagrangian-preserving surgeries $\ldots \ldots \ldots \ldots$

2 Conservation of the Blanchfield form 9

3 Relating Seifert matrices 11

4 Rational S-equivalence $\quad 14$

5 Relating integral Seifert matrices 17

6 Topological realization of matrix relations 21

7 Sequences of LP-surgeries 25

\section{Introduction}

\subsection{Context}

In GR04, Garoufalidis and Rozansky studied the rational vector space generated by the pairs $(M, K)$ modulo orientation-preserving homeomorphism, where $M$ is an integral homology 3-sphere (ZHS), that is an oriented compact 3-manifold which has the same homology with integral coefficients as $S^{3}$, and $K$ is a knot in $M$. They defined a filtration on this space by means of null-moves, that are surgeries on claspers (see Garoufalidis, Goussarov and Polyak [GGP01, and Habiro [Hab00]) whose leaves are trivial in $H_{1}(M \backslash$ $K ; \mathbb{Z})$. They studied this filtration with the Kricker lift of the Kontsevich integral defined in GK04. The first step in the study of this filtration is the determination of the classes of pairs $(M, K)$ up to null-moves. As a corollary of results of Matveev [Mat87, Naik and Stanford [NS03], and Trotter [Tro73], Garoufalidis and Rozansky established that two pairs $(M, K)$ as above can be obtained from one another by a finite sequence of nullmoves if and only if they admit S-equivalent Seifert matrices, and if and only if they have isomorphic integral Alexander modules and Blanchfield forms.

In this article, we consider pairs $(M, K)$, where $M$ is a rational homology 3-sphere $(\mathbb{Q H S})$, i.e. an oriented compact 3-manifold which has the same homology with rational coefficients as $S^{3}$, and $K$ is a null-homologous knot in $M$, i.e. a knot whose class in $H_{1}(M ; \mathbb{Z})$ is trivial. We define the null Lagrangian-preserving surgeries, which play the role played by the null-moves in the integral case. We prove that the classes of pairs $(M, K)$ modulo null Lagrangian-preserving surgeries are characterized by the classes of rational 
S-equivalence of their Seifert matrices, or by the isomorphism classes of their rational Alexander modules equipped with their Blanchfield forms. Furthermore, we prove that a fixed isomorphism between rational Alexander modules which preserves the Blanchfield form can be realized, up to multiplication by a power of $t$, by a finite sequence of null Lagrangian-preserving surgeries. Null Lagrangian-preserving surgeries define a filtration of the rational vector space generated by pairs $(M, K)$ modulo orientation-preserving homeomorphism. This article is a first step in the study of this filtration, that is useful in the study of equivariant finite type knot invariants.

In [GR04], Garoufalidis and Rozansky characterized the classes of pairs $(M, K)$, made of a $\mathbb{Z H S} M$ and a knot $K \subset M$, modulo null-moves, but they did not treat the question of the realization of a fixed isomorphism. In this article, we consider integral null Lagrangian-preserving surgeries, which generalize the null-moves, and define the same filtration of the vector space generated by all pairs $(M, K)$ modulo orientation-preserving homeomorphism. We prove that a fixed isomorphism between integral Alexander modules which preserves the Blanchfield form can be realized, up to multiplication by a power of $t$, by a finite sequence of integral null Lagrangian-preserving surgeries. Garoufalidis and Rozansky used their work to determine the graded space associated with the above filtration in the case of a trivial Alexander module. In order to study the general case of a maybe non trivial Alexander module, the realization result is essential.

When it does not seem to cause confusion, we use the same notation for a curve and its homology class.

\subsection{Alexander module and Blanchfield form}

We first recall the definition of the Alexander module and of the Blanchfield form. Let $(M, K)$ be a $\mathbb{Q} S K$-pair, that is a pair made of a rational homology sphere $M$ and a nullhomologous knot $K$ in $M$. Let $T(K)$ be a tubular neighborhood of $K$. The exterior of $K$ is $X=M \backslash \operatorname{Int}(T(K))$. Consider the natural projection $\pi: \pi_{1}(X) \rightarrow \frac{H_{1}(X ; \mathbb{Z})}{\text { torsion }} \cong \mathbb{Z}$, and the covering map $p: \tilde{X} \rightarrow X$ associated with its kernel. The covering $\tilde{X}$ is the infinite cyclic covering of $X$. The automorphism group of the covering, $A u t(\tilde{X})$, is isomorphic to $\mathbb{Z}$. It acts on $H_{1}(\tilde{X} ; \mathbb{Q})$. Denoting the action of a generator $\tau$ of $A u t(\tilde{X})$ as the multiplication by $t$, we get a structure of $\mathbb{Q}\left[t^{ \pm 1}\right]$-module on $\mathcal{A}(K)=H_{1}(\tilde{X} ; \mathbb{Q})$. This $\mathbb{Q}\left[t^{ \pm 1}\right]$-module is the Alexander module of $K$. It is a torsion $\mathbb{Q}\left[t^{ \pm 1}\right]$-module.

Definition 1.1. Let $(M, K)$ and $\left(M^{\prime}, K^{\prime}\right)$ be $\mathbb{Q S K}$-pairs. Let $\xi: \mathcal{A}(K) \rightarrow \mathcal{A}\left(K^{\prime}\right)$ be an isomorphism. The $\tau$-class of $\xi$ is the set of the isomorphisms $\xi \circ m_{k}$ for $k \in \mathbb{Z}$, where $m_{k}$ is the multiplication by $t^{k}$. 
Note that the $\tau$-class of $\xi$ is composed of all the isomorphisms that can be obtained from $\xi$ by composition by isomorphisms of $\mathcal{A}(K)$ or $\mathcal{A}\left(K^{\prime}\right)$ induced by automorphisms of the underlying coverings.

If $(M, K)$ is a $\mathbb{Z} S K$-pair, i.e. if $M$ is a $Z \mathrm{HS}$, define the integral Alexander module $\mathcal{A}_{\mathbb{Z}}(K)$ as the $\mathbb{Z}\left[t^{ \pm 1}\right]$-module $H_{1}(\tilde{X} ; \mathbb{Z})$, similarly. It is a torsion $\mathbb{Z}\left[t^{ \pm 1}\right]$-module, but we will see in Section 5 that it has no $\mathbb{Z}$-torsion. Hence it can be viewed as a $\mathbb{Z}\left[t^{ \pm 1}\right]$-submodule of $\mathcal{A}(K)$. Define as above the $\tau$-class of an isomorphism between integral Alexander modules.

On the Alexander module $\mathcal{A}(K)$, one can define the Blanchfield form, or equivariant linking pairing, $\phi_{K}: \mathcal{A}(K) \times \mathcal{A}(K) \rightarrow \frac{\mathbb{Q}(t)}{\mathbb{Q}\left[t^{ \pm 1}\right]}$, as follows. First define the equivariant linking number of two knots.

Definition 1.2. Let $J_{1}$ and $J_{2}$ be two knots in $\tilde{X}$ such that $J_{1} \cap \tau^{k}\left(J_{2}\right)=\emptyset$ for all $k \in \mathbb{Z}$. Let $\delta(t)$ be the annihilator of $\mathcal{A}(K)$. Then $\delta(\tau) J_{1}$ and $\delta(\tau) J_{2}$ are rationally null-homologous links. The equivariant linking number of $J_{1}$ and $J_{2}$ is

$$
l k_{e}\left(J_{1}, J_{2}\right)=\frac{1}{\delta(t) \delta\left(t^{-1}\right)} \sum_{k \in \mathbb{Z}} l k\left(\delta(\tau) J_{1}, \tau^{k}\left(\delta(\tau) J_{2}\right)\right) t^{k}
$$

One can easily see that $l k_{e}\left(J_{1}, J_{2}\right) \in \frac{1}{\delta(t)} \mathbb{Q}\left[t^{ \pm 1}\right], l k_{e}\left(J_{2}, J_{1}\right)(t)=l k_{e}\left(J_{1}, J_{2}\right)\left(t^{-1}\right)$, and $l k_{e}\left(P(\tau) J_{1}, Q(\tau) J_{2}\right)(t)=P(t) Q\left(t^{-1}\right) l k_{e}\left(J_{1}, J_{2}\right)(t)$. Now, if $\gamma$ (resp. $\eta$ ) is the homology class of $J_{1}$ (resp. $\left.J_{2}\right)$ in $\mathcal{A}(K)$, define $\phi_{K}(\gamma, \eta)$ by:

$$
\phi_{K}(\gamma, \eta)=l k_{e}\left(J_{1}, J_{2}\right) \bmod \mathbb{Q}\left[t^{ \pm 1}\right] .
$$

Extend $\phi_{K}$ to $\mathcal{A}(K) \times \mathcal{A}(K)$ by $\mathbb{Q}$-bilinearity. The Blanchfield form is hermitian:

$$
\phi_{K}(\gamma, \eta)(t)=\phi_{K}(\eta, \gamma)\left(t^{-1}\right) \quad \text { and } \quad \phi_{K}(P(t) \gamma, Q(t) \eta)(t)=P(t) Q\left(t^{-1}\right) \phi_{K}(\gamma, \eta)(t) \text {, }
$$

for all $\gamma, \eta \in \mathcal{A}(K)$ and all $P, Q \in \mathbb{Q}\left[t^{ \pm 1}\right]$. Moreover, it is non degenerate (see Blanchfield [Bla57]) : $\phi_{K}(\gamma, \eta)=0$ for all $\eta \in \mathcal{A}(K)$ implies $\gamma=0$.

\subsection{Seifert matrices}

Let $(M, K)$ be a $\mathbb{Q S K}$-pair. Let $\Sigma$ be a Seifert surface of $K$, i.e. a compact connected oriented surface in $M$ such that $\partial \Sigma=K$. Such a surface exists since $K$ is null-homologous. Let $g$ be the genus of $\Sigma$. Let $\left(f_{i}\right)_{1 \leq i \leq 2 g}$ be a symplectic basis of $H_{1}(\Sigma ; \mathbb{Z})$, i.e. a basis such that the matrix of the intersection form in $\left(f_{i}\right)_{1 \leq i \leq 2 g}$ is $-J$, where $J$ is made of blocks $\left(\begin{array}{cc}0 & -1 \\ 1 & 0\end{array}\right)$ on the diagonal, and zeros elsewhere. The Seifert matrix of $K$ associated with $\Sigma$ 
and $\left(f_{i}\right)_{1 \leq i \leq 2 g}$ is the matrix $V \in \mathcal{M}_{2 g}(\mathbb{Q})$ defined by $V_{i j}=l k\left(f_{i}, f_{j}^{+}\right)$, where $f_{j}^{+}$is a pushoff of $f_{j}$ in the direction of the positive normal of $\Sigma$. This matrix satisfies $V-V^{t}=J$, where $V^{t}$ denotes the transpose of $V$. Any rational (resp. integral) matrix with this property will be called a Seifert matrix (resp. an integral Seifert matrix). In Section 6, we prove that any such matrix is indeed the Seifert matrix of a $\mathbb{Q S K}$-pair $(M, K)$, and the Seifert matrix of a $\mathbb{Z S K}$-pair if the matrix is integral.

Given the Seifert matrix $V$, one can compute the Alexander module $\mathcal{A}(K)$ and the Blanchfield form $\phi_{K}$. Construct a surface $\hat{\Sigma}$ by adding a band to $\Sigma$ along $K$, so that $\hat{\Sigma}$ is homeomorphic to $\Sigma$ and contains $\Sigma$ and $K$ in its interior. Let $T(\Sigma)=\hat{\Sigma} \times[-1,1]$ be a tubular neighborhood of $\Sigma$. For $1 \leq i \leq 2 g$, let $e_{i} \subset(\operatorname{Int}(T(\Sigma)) \backslash \Sigma)$ be a meridian of $f_{i}$. The module $\mathcal{A}(K)$ can be presented as:

$$
\mathcal{A}(K)=\frac{\bigoplus_{1 \leq i \leq 2 g} \mathbb{Q}\left[t^{ \pm 1}\right] b_{i}}{\bigoplus_{1 \leq j \leq 2 g} \mathbb{Q}\left[t^{ \pm 1}\right] \partial S_{j}}
$$

where the $b_{i}$ are lifts of the $e_{i}$ in the infinite cyclic covering $\tilde{X}$, and the $S_{j}$ are lifts of the $f_{j} \times[-1,1]$. Set $f_{j}^{+}=f_{j} \times\{1\}$ and $f_{j}^{-}=f_{j} \times\{-1\}$. Assume the $b_{i}$ are all chosen in the same copy of $M \backslash \Sigma$. For $1 \leq j \leq 2 g$, let $\tilde{f}_{j}^{+}$and $\tilde{f}_{j}^{-}$be lifts of $f_{j}^{+}$and $f_{j}^{-}$in the same copy of $M \backslash \Sigma$ as the $b_{i}$. Assume the $S_{j}$ are chosen so that $\partial S_{j}=t \tilde{f}_{j}^{+}-\tilde{f}_{j}^{-}$. Then $\partial S_{j}=$ $\sum_{1 \leq i \leq 2 g}\left(t V-V^{t}\right)_{i j} b_{i}$, hence $t V-V^{t}$ is a presentation matrix of $\mathcal{A}(K)$ (see [Lic97, Chapter $6])$. Moreover, we have $l k_{e}\left(\partial S_{j}, b_{k}\right)=(1-t) \delta_{k j}$. Using the expression of $\partial S_{j}$ in terms of the $b_{i}$, it follows that the form $\phi_{K}$ is given by $\phi_{K}\left(b_{i}, b_{k}\right)=(1-t)\left(\left(t V-V^{t}\right)^{-1}\right)_{k i} \bmod \mathbb{Q}\left[t^{ \pm 1}\right]$ (see Kearton [Kea75, §8]). If $(M, K)$ is a $\mathbb{Z}$ SK-pair, then $V$ is integral, and the same construction shows that $t V-V^{t}$ is a presentation matrix of the $\mathbb{Z}\left[t^{ \pm 1}\right]$-module $\mathcal{A}_{\mathbb{Z}}(K)$.

A $\mathbb{Q} S K$-system is a quintuple $(M, K, \Sigma, f, V)$ where $(M, K)$ is a $\mathbb{Q S K}$-pair, $\Sigma$ is a Seifert surface of $K$ in $M, \underline{f}=\left(f_{i}\right)_{1 \leq i \leq 2 g}$ is a symplectic basis of $H_{1}(\Sigma ; \mathbb{Z})$, and $V$ is the associated Seifert matrix. Given a $\mathbb{Q} S K$-system, the associated family $\left(b_{i}\right)_{1 \leq i \leq 2 g}$ of generators of $\mathcal{A}(K)$ is determined up to multiplication of the whole family by $t^{k}$ for some integer $k$. A $\mathbb{Z} S K$-system $(M, K, \Sigma, \underline{f}, V)$ is a $\mathbb{Q S K}$-system such that $M$ is a $\mathbb{Z H S}$.

\subsection{S-equivalence}

Definition 1.3. A row enlargement of a matrix $V \in \mathcal{M}_{2 g}(\mathbb{Q})$ is a matrix $W=\left(\begin{array}{ccc}0 & 0 & 0 \\ 1 & x & \rho^{t} \\ 0 & \rho & V\end{array}\right)$, where $x \in \mathbb{Q}$ and $\rho \in \mathbb{Q}^{2 g}$. Then the matrix $V$ is a row reduction of $W$. A column 
enlargement of $V$ is a matrix $W=\left(\begin{array}{ccc}0 & -1 & 0 \\ 0 & x & \rho^{t} \\ 0 & \rho & V\end{array}\right)$, where $x \in \mathbb{Q}$ and $\rho \in \mathbb{Q}^{2 g}$. Then the matrix $V$ is a column reduction of $W$. If all the coefficients of the matrices $V$ and $W$ are integers, then the enlargement, or the reduction, is said to be integral.

Note that an enlargement or a reduction of a Seifert matrix still is a Seifert matrix. An enlargement of a Seifert matrix corresponds to the addition of a tube to the Seifert surface.

Definition 1.4. A matrix $P \in \mathcal{M}_{2 g}(\mathbb{Q})$ is symplectic if $P J P^{t}=J$. A rational (resp. integral) symplectic congruence from a matrix $V$ to a matrix $V^{\prime}$ is an equality $V^{\prime}=P V P^{t}$ for some rational (resp. integral) symplectic matrix $P$.

It is more usual to define a symplectic matrix by $P^{t} J P=J$. However, the two definitions are equivalent, and our choice takes sense when we interpret the symplectic matrix involved in a congruence relation beetween Seifert matrices as the matrix of the corresponding isomorphism beetween Alexander modules, see Proposition 3.1 .

Note that, since a symplectic matrix has determinant 1, a symplectic rational (resp. integral) matrix is invertible over $\mathbb{Q}($ resp. $\mathbb{Z})$.

Definition 1.5. An elementary rational S-equivalence is an enlargement, a reduction, or a rational symplectic congruence. Two Seifert matrices are rationally S-equivalent if they can be obtained from one another by a finite sequence of elementary rational Sequivalences.

In particular, two Seifert matrices of a $\mathbb{Q S K}$-pair $(M, K)$ are rationally S-equivalent (see [Lic97, Theorem 8.4] for the integral case, which easily generalizes). In Section 2, we show that, given two $\mathbb{Q S K}$-systems, a rational S-equivalence between their Seifert matrices induces a canonical $\tau$-class of isomorphisms between their Alexander modules preserving the Blanchfield form. In Section 3, we prove the converse:

Proposition 1.6. Let $(M, K, \Sigma, f, V)$ and $\left(M^{\prime}, K^{\prime}, \Sigma^{\prime}, f^{\prime}, V^{\prime}\right)$ be two $\mathbb{Q} S K$-systems. Let $\xi: \mathcal{A}(K) \rightarrow \mathcal{A}\left(K^{\prime}\right)$ be an isomorphism which preserves the Blanchfield form. Then $V$ and $V^{\prime}$ are related by a rational $S$-equivalence which canonically induces the $\tau$-class of $\xi$.

Definition 1.7. Two Seifert matrices are semi-integrally S-equivalent if they can be obtained from one another by a finite sequence of enlargements, reductions, and integral symplectic congruences.

In Section 4, as a consequence of Lemmas 4.1 and 4.2, we obtain: 
Theorem 1.8. Two Seifert matrices are rationally $S$-equivalent if and only if they are semi-integrally S-equivalent. Furthermore, let $(M, K, \Sigma, \underline{f}, V)$ and $\left(M^{\prime}, K^{\prime}, \Sigma^{\prime}, f^{\prime}, V^{\prime}\right)$ be two $\mathbb{Q} S K$-systems. Let $\xi: \mathcal{A}(K) \rightarrow \mathcal{A}\left(K^{\prime}\right)$ be an isomorphism which preserves the Blanchfield form. Then $V$ and $V^{\prime}$ are related by a semi-integral S-equivalence which canonically induces the $\tau$-class of $\xi$.

In the case of $\mathbb{Z S K}$-systems, for later applications, we need results with only integral coefficients.

Definition 1.9. Two Seifert matrices are integrally S-equivalent if they can be obtained from one another by a finite sequence of integral enlargements, integral reductions, and integral symplectic congruences.

In Section 2, we prove that, given two $\mathbb{Z} S K$-systems, an integral S-equivalence between their Seifert matrices induces a canonical $\tau$-class of isomorphisms between their integral Alexander modules preserving the Blanchfield form. In Section 5 , we prove:

Theorem 1.10. Let $(M, K, \Sigma, \underline{f}, V)$ and $\left(M^{\prime}, K^{\prime}, \Sigma^{\prime}, f^{\prime}, V^{\prime}\right)$ be two $\mathbb{Z} S K$-systems. Let $\xi: \mathcal{A}_{\mathbb{Z}}(K) \rightarrow \mathcal{A}_{\mathbb{Z}}\left(K^{\prime}\right)$ be an isomorphism which preserves the Blanchfield form. Then $V$ and $V^{\prime}$ are related by an integral $S$-equivalence which canonically induces the $\tau$-class of $\xi$.

\subsection{Lagrangian-preserving surgeries}

Definition 1.11. For $g \in \mathbb{N}$, a genus g rational (resp. integral) homology handlebody $(\mathbb{Q H H}$, resp. $\mathbb{Z H H})$ is a 3-manifold which is compact, oriented, and which has the same homology with rational (resp. integral) coefficients as the standard genus $g$ handlebody.

Such a $\mathbb{Q H H}$ is connected, and its boundary is necessarily homeomorphic to the standard genus $g$ surface. Note that a $\mathbb{Z H H}$ is a $\mathbb{Q H H}$.

Definition 1.12. The Lagrangian $\mathcal{L}_{A}$ of a $\mathbb{Q H H} A$ is the kernel of the map

$$
i_{*}: H_{1}(\partial A ; \mathbb{Q}) \rightarrow H_{1}(A ; \mathbb{Q})
$$

induced by the inclusion. Two $\mathbb{Q H H}$ 's $A$ and $B$ have $L P$-identified boundaries if $(A, B)$ is equipped with a homeomorphism $h: \partial A \stackrel{\cong}{\longrightarrow} \partial B$ such that $h_{*}\left(\mathcal{L}_{A}\right)=\mathcal{L}_{B}$.

The Lagrangian of a $\mathbb{Q} H H A$ is indeed a Lagrangian subspace of $H_{1}(\partial A ; \mathbb{Q})$ with respect to the intersection form. 
Let $M$ be a $\mathbb{Q H S}$, let $A \subset M$ be a $\mathbb{Q H H}$, and let $B$ be a $\mathbb{Q H H}$ whose boundary is LP-identified with $\partial A$. Set $M\left(\frac{B}{A}\right)=(M \backslash \operatorname{Int}(A)) \cup_{\partial A=\partial B} B$. We say that the $\mathbb{Q} H S M\left(\frac{B}{A}\right)$ is obtained from $M$ by Lagrangian-preserving surgery, or LP-surgery.

Given a $\mathbb{Q S K}$-pair $(M, K)$, a null- $\mathbb{Q} H H$ in $M \backslash K$ is a $\mathbb{Q H H} A \subset M \backslash K$ such that the map $i_{*}: H_{1}(A ; \mathbb{Q}) \rightarrow H_{1}(M \backslash K ; \mathbb{Q})$ induced by the inclusion has a trivial image. A null $L P$-surgery on $(M, K)$ is an LP-surgery $\left(\frac{B}{A}\right)$ such that $A$ is null in $M \backslash K$. The $\mathbb{Q}$ SK-pair obtained by surgery is denoted by $(M, K)\left(\frac{B}{A}\right)$.

Similarly, define integral LP-surgeries, null-ZZHH's, and integral null LP-surgeries. The null-moves introduced by Garoufalidis and Rozansky in [GR04 are defined as null Borromean surgeries. Borromean surgeries are specific integral LP-surgeries (see Matveev [Mat87]). In [AL05, Lemma 4.11], Auclair and Lescop proved that two $\mathbb{Z} H H$ 's whose boundaries are LP-identified can be obtained from one another by a finite sequence of Borromean surgeries in the interior of the $\mathbb{Z H H}$ 's. Hence the classes of $\mathbb{Z} S K$-pairs modulo null integral LP-surgeries are exactly the classes of $\mathbb{Z S K}$-pairs modulo null-moves.

In Section 2, we prove that a null LP-surgery induces a canonical isomorphism from the Alexander module of the initial $\mathbb{Q S K}$-pair to the Alexander module of the surgered $\mathbb{Q}$ SK-pair, which preserves the Blanchfield form. Conversely, in Section 6, we prove:

Theorem 1.13. Let $(M, K)$ and $\left(M^{\prime}, K^{\prime}\right)$ be $\mathbb{Q} S K$-pairs. Assume there is an isomorphism $\xi: \mathcal{A}(K) \rightarrow \mathcal{A}\left(K^{\prime}\right)$ which preserves the Blanchfield form. Then $\left(M^{\prime}, K^{\prime}\right)$ can be obtained from $(M, K)$ by a finite sequence of null LP-surgeries which induces an isomorphism in the $\tau$-class of $\xi$.

Similarly, in Section 2, we prove that an integral null LP-surgery induces a canonical isomorphism from the integral Alexander module of the initial $\mathbb{Z S K}$-pair to the Alexander module of the surgered $\mathbb{Z}$ SK-pair, which preserves the Blanchfield form. In Section 6, we prove:

Theorem 1.14. Let $(M, K)$ and $\left(M^{\prime}, K^{\prime}\right)$ be $\mathbb{Z} S K$-pairs. Assume there is an isomorphism $\xi: \mathcal{A}_{\mathbb{Z}}(K) \rightarrow \mathcal{A}_{\mathbb{Z}}\left(K^{\prime}\right)$ which preserves the Blanchfield form. Then $\left(M^{\prime}, K^{\prime}\right)$ can be obtained from $(M, K)$ by a finite sequence of integral null LP-surgeries which induces an isomorphism in the $\tau$-class of $\xi$.

We end the article by proving the following proposition in Section 7 .

Proposition 1.15. There are $\mathbb{Q} S K$-pairs $(M, K)$ and $\left(M^{\prime}, K^{\prime}\right)$ that can be obtained from one another by a finite sequence of null LP-surgeries, but not by a single null LP-surgery.

Note that this cannot happen in the case of integral null LP-surgeries. Indeed, as mentioned above, these surgeries can be realized by Borromean surgeries, which can be realized in the regular neighborhood of graphs. 
Acknowledgements I would like to sincerely thank my advisor, Christine Lescop, for her great guidance.

\section{Conservation of the Blanchfield form}

In this section, we prove that null LP-surgeries (Lemma 2.1) and relations of rational Sequivalence (Lemma 2.3) induce canonical $\tau$-classes of isomorphisms between the Alexander modules which preserve the Blanchfield form. We also state similar results in the integral case.

Lemma 2.1. Let $(M, K)$ be a $\mathbb{Q} S K$-pair. Let $A$ be a null- $\mathbb{Q} H H$ in $M \backslash K$. Let $B$ be a $\mathbb{Q} H H$ whose boundary is LP-identified with $\partial A$. Set $\left(M^{\prime}, K^{\prime}\right)=(M, K)\left(\frac{B}{A}\right)$. Then the surgery induces a canonical isomorphism $\xi: \mathcal{A}(K) \rightarrow \mathcal{A}\left(K^{\prime}\right)$ which preserves the Blanchfield form.

Proof. In this proof, the homology modules are considered with rational coefficients. Let $\tilde{X}$ (resp. $\left.\tilde{X}^{\prime}\right)$ be the infinite cyclic covering associated with $(M, K)\left(\operatorname{resp} .\left(M^{\prime}, K^{\prime}\right)\right)$. The preimage $\tilde{A}$ of $A$ in $\tilde{X}$ (resp. $\tilde{B}$ of $B$ in $\tilde{X}^{\prime}$ ) is the disjoint union of $\mathbb{Z}$ copies $A_{i}$ of $A$ (resp. $B_{i}$ of $\left.B\right)$.

Set $Y=\tilde{X} \backslash \operatorname{Int}(\tilde{A})$. The Mayer-Vietoris sequence associated with $\tilde{X}=\tilde{A} \cup Y$ yields the exact sequence:

$$
H_{1}(\partial \tilde{A}) \rightarrow H_{1}(\tilde{A}) \oplus H_{1}(Y) \rightarrow H_{1}(\tilde{X}) \rightarrow 0 .
$$

Since $H_{1}(\partial \tilde{A}) \cong H_{1}(\tilde{A}) \oplus\left(\mathbb{Q}\left[t^{ \pm 1}\right] \otimes \mathcal{L}_{A}\right)$, we get $H_{1}(\tilde{X}) \cong \frac{H_{1}(Y)}{\mathbb{Q}\left[t^{ \pm 1}\right] \otimes \mathcal{L}_{A}}$. Similarly, $H_{1}\left(\tilde{X}^{\prime}\right) \cong$ $\frac{H_{1}(Y)}{\mathbb{Q}\left[t^{ \pm 1}\right] \otimes \mathcal{L}_{B}}$. Since $\mathcal{L}_{A}=\mathcal{L}_{B}$, the Alexander modules $H_{1}(\tilde{X})$ and $H_{1}\left(\tilde{X}^{\prime}\right)$ are canonically identified.

Now consider two null-homologous knots $J$ and $J^{\prime}$ in $\tilde{X}$ that do not meet $\tilde{A}$, and such that $J \cap \tau^{k}\left(J^{\prime}\right)=\emptyset$ for all $k \in \mathbb{Z}$. Consider a Seifert surface $\Sigma$ of $J$. Assume that $\Sigma$ is transverse to $\partial \tilde{A}$ and $J^{\prime}$. Write $\Sigma=\Sigma_{1} \cup \Sigma_{2}$, where $\Sigma_{1}=\Sigma \cap Y$ and $\Sigma_{2}=\Sigma \cap \tilde{A}$. Since $J^{\prime}$ does not meet $\Sigma_{2}$, the linking number $l k_{\tilde{X}}\left(J, J^{\prime}\right)$ is equal to the algebraic intersection number $<J^{\prime}, \Sigma_{1}>$. Now $\partial \Sigma_{2}$ is an integral linear combination of curves $\alpha_{i} \in \mathcal{L}_{A_{i}}$. In $\tilde{X}^{\prime}$, each $\alpha_{i}$ lies in $\mathcal{L}_{B_{i}}$, so each $\alpha_{i}$ has a multiple that bounds a surface in $B_{i}$. Thus, there is a surface $\Sigma_{3} \subset \tilde{B}$ such that $\partial \Sigma_{3}=n \partial \Sigma_{2}$ for some integer $n$. We have $n J=\partial\left(n \Sigma_{1} \cup \Sigma_{3}\right)$, thus:

$$
l k_{\tilde{X}^{\prime}}\left(J, J^{\prime}\right)=\frac{1}{n}<J^{\prime}, n \Sigma_{1} \cup \Sigma_{3}>=<J^{\prime}, \Sigma_{1}>=l k_{\tilde{X}}\left(J, J^{\prime}\right) .
$$


Since any class $\gamma$ in $H_{1}(\tilde{X})$ has a multiple that can be represented by a $\operatorname{knot} J$ in $Y$ such that $P(t) . J$ is null-homologous for some $P \in \mathbb{Q}\left[t^{ \pm 1}\right]$, the Blanchfield form is preserved.

The previous proof still works, when $\mathbb{Q}$ is replaced by $\mathbb{Z}$. Therefore:

Lemma 2.2. Let $(M, K)$ and $\left(M^{\prime}, K^{\prime}\right)$ be $\mathbb{Z} S K$-pairs. Assume $\left(M^{\prime}, K^{\prime}\right)$ can be obtained from $(M, K)$ by an integral null LP-surgery. Then this surgery induces a canonical isomorphism between their integral Alexander modules which preserves the Blanchfield form.

Lemma 2.3. Let $(M, K, \Sigma, f, V)$ and $\left(M^{\prime}, K^{\prime}, \Sigma^{\prime}, f^{\prime}, V^{\prime}\right)$ be $\mathbb{Q}$ SK-systems. If $V$ and $V^{\prime}$ are rationally $S$-equivalent, then any $S$-equivalence from $V$ to $V^{\prime}$ induces a canonical $\tau$ class of isomorphisms from $\mathcal{A}(K)$ to $\mathcal{A}\left(K^{\prime}\right)$ which preserve the Blanchfield form.

Proof. Let $\left(b_{i}\right)_{1 \leq i \leq 2 g}$ be a family of generators of $\mathcal{A}(K)$ associated with $V$. Set $W=$ $t V-V^{t}$. Recall the Blanchfield form $\phi_{K}$ is given by $\phi_{K}\left(b_{i}, b_{j}\right)=(1-t)\left(W^{-1}\right)_{j i}$. Set $b=\left(\begin{array}{llll}b_{1} & b_{2} & \ldots & b_{2 g}\end{array}\right)$, and $r=\left(\begin{array}{llll}r_{1} & r_{2} & \ldots & r_{2 g}\end{array}\right)=b W$. We have:

$$
\mathcal{A}(K)=\frac{\bigoplus_{1 \leq i \leq 2 g} \mathbb{Q}\left[t^{ \pm 1}\right] b_{i}}{\bigoplus_{1 \leq j \leq 2 g} \mathbb{Q}\left[t^{ \pm 1}\right] r_{j}} .
$$

Define the same notation with primes for the $\mathbb{Q S K}$-pair $\left(M^{\prime}, K^{\prime}\right)$.

First assume that $V^{\prime}=P V P^{t}$ for a rational symplectic matrix $P$. Note that $W^{\prime}=$ $P W P^{t}$. Define a $\mathbb{Q}\left[t^{ \pm 1}\right]$-isomorphism:

$$
\begin{array}{clc}
\tilde{\xi}: \bigoplus_{1 \leq i \leq 2 g} \mathbb{Q}\left[t^{ \pm 1}\right] b_{i} & \rightarrow & \bigoplus_{1 \leq i \leq 2 g} \mathbb{Q}\left[t^{ \pm 1}\right] b_{i}^{\prime} \\
b_{i} & \mapsto & \left(b^{\prime} P\right)_{i}
\end{array}
$$

We have $\tilde{\xi}\left(r_{j}\right)=\left(b^{\prime} P W\right)_{j}=\left(r^{\prime}\left(P^{t}\right)^{-1}\right)_{j}$, thus $\tilde{\xi}\left(\bigoplus_{1 \leq j \leq 2 g} \mathbb{Q}\left[t^{ \pm 1}\right] r_{j}\right)=\bigoplus_{1 \leq j \leq 2 g} \mathbb{Q}\left[t^{ \pm 1}\right] r_{j}^{\prime}$. Hence $\tilde{\xi}$ induces an isomorphism $\xi: \mathcal{A}(K) \rightarrow \mathcal{A}\left(K^{\prime}\right)$. Now, we have:

$$
\begin{aligned}
\phi_{K^{\prime}}\left(\xi\left(b_{i}\right), \xi\left(b_{j}\right)\right) & =\phi_{K^{\prime}}\left(\left(b^{\prime} P\right)_{i},\left(b^{\prime} P\right)_{j}\right) \\
& =\sum_{k, l} p_{k i} p_{l j}(1-t)\left(\left(W^{\prime}\right)^{-1}\right)_{l k} \\
& =(1-t)\left(P^{t}\left(\left(P^{t}\right)^{-1} W^{-1} P^{-1}\right) P\right)_{j i} \\
& =\phi_{K}\left(b_{i}, b_{j}\right) .
\end{aligned}
$$

It remains to treat the case of an enlargement. Assume $V=\left(\begin{array}{ccc}0 & 0 & 0 \\ 1 & x & \rho^{t} \\ 0 & \rho & V^{\prime}\end{array}\right)$. Then:

$$
W=\left(\begin{array}{ccc}
0 & -1 & 0 \\
t & x(t-1) & (t-1) \rho^{t} \\
0 & (t-1) \rho & W^{\prime}
\end{array}\right)
$$


Thus $b_{2}$ is trivial, and $b_{1}$ is a linear combination over $\mathbb{Q}\left[t^{ \pm 1}\right]$ of the $b_{i}$ for $3 \leq i \leq 2 g$. Hence there is an isomorphism $\left(\mathcal{A}(K), \phi_{K}\right) \cong\left(\mathcal{A}\left(K^{\prime}\right), \phi_{K^{\prime}}\right)$ which identifies $b_{i}$ with $b_{i-2}^{\prime}$ for $3 \leq i \leq 2 g$. Proceed similarly for a column enlargement.

Since the families $\left(b_{i}\right)_{1 \leq i \leq 2 g}$ and $\left(b_{i}^{\prime}\right)_{1 \leq i \leq 2 g}$ are determined up to multiplication by a power of $t$, we have associated a $\tau$-class of isomorphisms to each elementary S-equivalence. For a general rational S-equivalence, just compose the $\tau$-classes associated with the elementary S-equivalences.

The previous proof still works, when $\mathbb{Q}$ is replaced by $\mathbb{Z}$. Therefore:

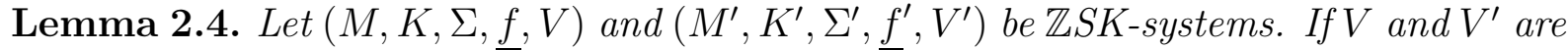
integrally $S$-equivalent, then any integral $S$-equivalence from $V$ to $V^{\prime}$ induces a canonical $\tau$-class of isomorphisms from $\mathcal{A}_{\mathbb{Z}}(K)$ to $\mathcal{A}_{\mathbb{Z}}\left(K^{\prime}\right)$ which preserve the Blanchfield form.

\section{Relating Seifert matrices}

In this section, we prove the next proposition, which implies Proposition 1.6 for invertible Seifert matrices $V$ and $V^{\prime}$. We end the section by deducing the general case.

Proposition 3.1. Let $(M, K, \Sigma, \underline{f}, V)$ and $\left(M^{\prime}, K^{\prime}, \Sigma^{\prime}, f^{\prime}, V^{\prime}\right)$ be two $\mathbb{Q} S K$-systems. Let $\xi: \mathcal{A}(K) \rightarrow \mathcal{A}\left(K^{\prime}\right)$ be an isomorphism which preserves the Blanchfield form. Let $\underline{b}=\left(b_{i}\right)_{1 \leq i \leq 2 g}$ (resp. $\underline{b}^{\prime}=\left(b_{i}^{\prime}\right)_{1 \leq i \leq 2 g}$ ) be a family of generators of $\mathcal{A}(K)$ (resp. $\mathcal{A}\left(K^{\prime}\right)$ ) associated with $V$ (resp. $\left.V^{\prime}\right)$. If $V$ and $V^{\prime}$ are invertible, then $(\underline{b})$ and $\left(\underline{b}^{\prime}\right)$ are $\mathbb{Q}$-bases of $\mathcal{A}(K)$ and $\mathcal{A}\left(K^{\prime}\right)$ respectively. Let $P$ be the matrix of $\xi$ with respect to the bases $(\underline{b})$ and $\left(\underline{b}^{\prime}\right)$. Then $P$ is symplectic and $V^{\prime}=P V P^{t}$.

This proposition specifies a result of Trotter [Tro73, Proposition 2.12].

Lemma 3.2. Let $(M, K, \Sigma, f, V)$ be a $\mathbb{Q} S K$-system. Let $\underline{b}=\left(b_{i}\right)_{1 \leq i \leq 2 g}$ be a family of generators of $\mathcal{A}(K)$ associated with $V$. If $V$ is invertible, then $\underline{b}$ is a $\mathbb{Q}$-basis of $\mathcal{A}(K)$, and the action of $t$ is given by the matrix $V^{t} V^{-1}$ with respect to the basis $\underline{b}$.

Proof. We have:

$$
\mathcal{A}(K)=\frac{\bigoplus_{1 \leq i \leq 2 g} \mathbb{Q}\left[t^{ \pm 1}\right] b_{i}}{\bigoplus_{1 \leq j \leq 2 g} \mathbb{Q}\left[t^{ \pm 1}\right] r_{j}},
$$

where $r_{j}=\sum_{1 \leq i \leq 2 g}\left(t V-V^{t}\right)_{i j} b_{i}$. Represent the elements of $\left(\mathbb{Q}\left[t^{ \pm 1}\right]\right)^{2 g}=\bigoplus_{1 \leq i \leq 2 g} \mathbb{Q}\left[t^{ \pm 1}\right] b_{i}$ (resp. $\mathbb{Q}^{2 g}$ ) by column vectors giving their coordinates in the basis $\underline{b}$ (resp. in the canonical 
basis). Define a $\mathbb{Q}$-linear map $\varphi:\left(\mathbb{Q}\left[t^{ \pm 1}\right]\right)^{2 g} \rightarrow \mathbb{Q}^{2 g}$ by $t^{k} X \mapsto\left(V^{t} V^{-1}\right)^{k} X$ for all vector $X$ with rational coefficients. Let us prove that $\varphi$ induces a $\mathbb{Q}$-isomorphism from $\mathcal{A}(K)$ to $\mathbb{Q}^{2 g}$.

It is easy to see that $\mathbb{Q}\left[t^{ \pm 1}\right] r_{j} \subset \operatorname{ker}(\varphi)$ for all $j$. Let $u \in \operatorname{ker}(\varphi)$. Write $u=\sum_{p \leq k \leq q} t^{k} X_{k}$, where the $X_{k}$ are column vectors with rational coefficients. Since $\sum_{p \leq k \leq q}\left(V^{t} V^{-1}\right)^{k} X_{k}=$ 0 , we have $X_{p}=-\sum_{p<k \leq q}\left(V^{t} V^{-1}\right)^{k-p} X_{k}$. Thus:

$$
\begin{aligned}
u & =\sum_{p<k \leq q}\left(t^{k} X_{k}-t^{p}\left(V^{t} V^{-1}\right)^{k-p} X_{k}\right) \\
& =\left(t V-V^{t}\right) . \sum_{p<k \leq q} \sum_{p<i \leq k} t^{i-1}\left(V^{-1} V^{t}\right)^{k-i} V^{-1} X_{k}
\end{aligned}
$$

Hence $u \in \bigoplus_{1 \leq j \leq 2 g} \mathbb{Q}\left[t^{ \pm 1}\right] r_{j}$, and it follows that $\operatorname{ker}(\varphi)=\bigoplus_{1 \leq j \leq 2 g} \mathbb{Q}\left[t^{ \pm 1}\right] r_{j}$.

Corollary 3.3. Let $(M, K, \Sigma, f, V)$ be a $\mathbb{Q} S K$-system. Let $\underline{b}=\left(b_{i}\right)_{1 \leq i \leq 2 g}$ be a family of generators of $\mathcal{A}(K)$ associated with $V$. Assume $V$ is invertible. Let $T$ be the matrix of the multiplication by $t$ in the basis $\underline{b}$. Then $V=\left(I_{2 g}-T\right)^{-1} J$.

Proof. By Lemma 3.2, $T=V^{t} V^{-1}$. Hence $(I-T) V=V-V^{t}=J$.

Consider $\mathbb{Q}(t)$ as the direct sum over $\mathbb{Q}$ of $\Lambda=\mathbb{Q}\left[t, t^{-1},(1-t)^{-1}\right]$ and the subspace $\mathcal{E}$ consisting of 0 and all proper fractions with denominator prime to $t$ and $(1-t)$, where proper means that the degree of the fraction numerator is strictly lower than the degree of the denominator. Define a $\mathbb{Q}$-linear function $\chi$ on $\mathbb{Q}(t)$ by $\chi(E)=E^{\prime}(1)$ if $E \in \mathcal{E}$ and $\chi(E)=0$ if $E \in \Lambda$. Since $\chi$ vanishes on $\mathbb{Q}\left[t^{ \pm 1}\right]$, we may also consider it as a function on $\frac{\mathbb{Q}(t)}{\mathbb{Q}\left[t^{ \pm 1}\right]}$.

Lemma 3.4. For $E \in \mathcal{E}, \chi((t-1) E)=E(1)$.

Proof. If $F \in \mathbb{Q}(t)$ has denominator prime to $t$ and $1-t$ and numerator of degree less than or equal to the degree of its denominator, then $F$ can be written as the sum of a rational constant and of an element of $\mathcal{E}$. Hence $\chi(F)=F^{\prime}(1)$. Apply this to $F=(t-1) E$.

Lemma 3.5. Let $(M, K, \Sigma, f, V)$ be a $\mathbb{Q} S K$-system. Let $\left(b_{i}\right)_{1 \leq i \leq 2 g}$ be a family of generators of $\mathcal{A}(K)$ associated with $V$. Define a matrix $S$ by $S_{i j}=\chi\left(\phi_{K}\left(b_{j}, b_{i}\right)\right)$. Then $S=-\left(V-V^{t}\right)^{-1}=J$.

Proof. We have $S_{i j}=-\chi\left((t-1)\left(\left(t V-V^{t}\right)^{-1}\right)_{i j}\right)$. Let $\Delta(t)=\operatorname{det}\left(t V-V^{t}\right)$ be the Alexander polynomial of $(M, K)$. Then $\Delta(1) \neq 0$, and since $V$ is invertible, $\Delta(0) \neq 0$ and the degree of $\Delta$ is equal to the size of the matrix $V$. Hence, by Lemma 3.4, $S_{i j}=$ $-\left(\left(t V-V^{t}\right)^{-1}\right)_{i j}(1)$. Conclude with $V-V^{t}=J$ and $J^{-1}=-J$. 
Proof of Proposition 3.1. Let $T$ (resp. $T^{\prime}$ ) be the matrix of the action of $t$ in the basis $\underline{b}$ (resp. $\left.\underline{b}^{\prime}\right)$. By Corollary 3.3, $V=\left(I_{2 g}-T\right)^{-1} J$ and $V^{\prime}=\left(I_{2 g}-T^{\prime}\right)^{-1} J$. Since $\xi$ preserves the action of $t$, we have $P T=T^{\prime} P$. Since $\xi$ preserves the Blanchfield form, Lemma 3.5 implies $J=P^{t} J P$. It follows that $V^{\prime}=P V P^{t}$.

Lemma 3.6. For any matrix $V \in \mathcal{M}_{2 g}(\mathbb{Q})$, with $g>0$, satisfying $V-V^{t}=J$, there exists a $\mathbb{Q} S K$-pair $(M, K)$ that admits $V$ as a Seifert matrix. If $V \in \mathcal{M}_{2 g}(\mathbb{Z})$, then $M$ can be chosen to be $S^{3}$.

Proof. Set $V=\left(v_{i j}\right)_{1 \leq i, j \leq 2 g}$. By [Mou12b, Corollary 2.13], there is a $\mathbb{Q H S} M$ and pairwise
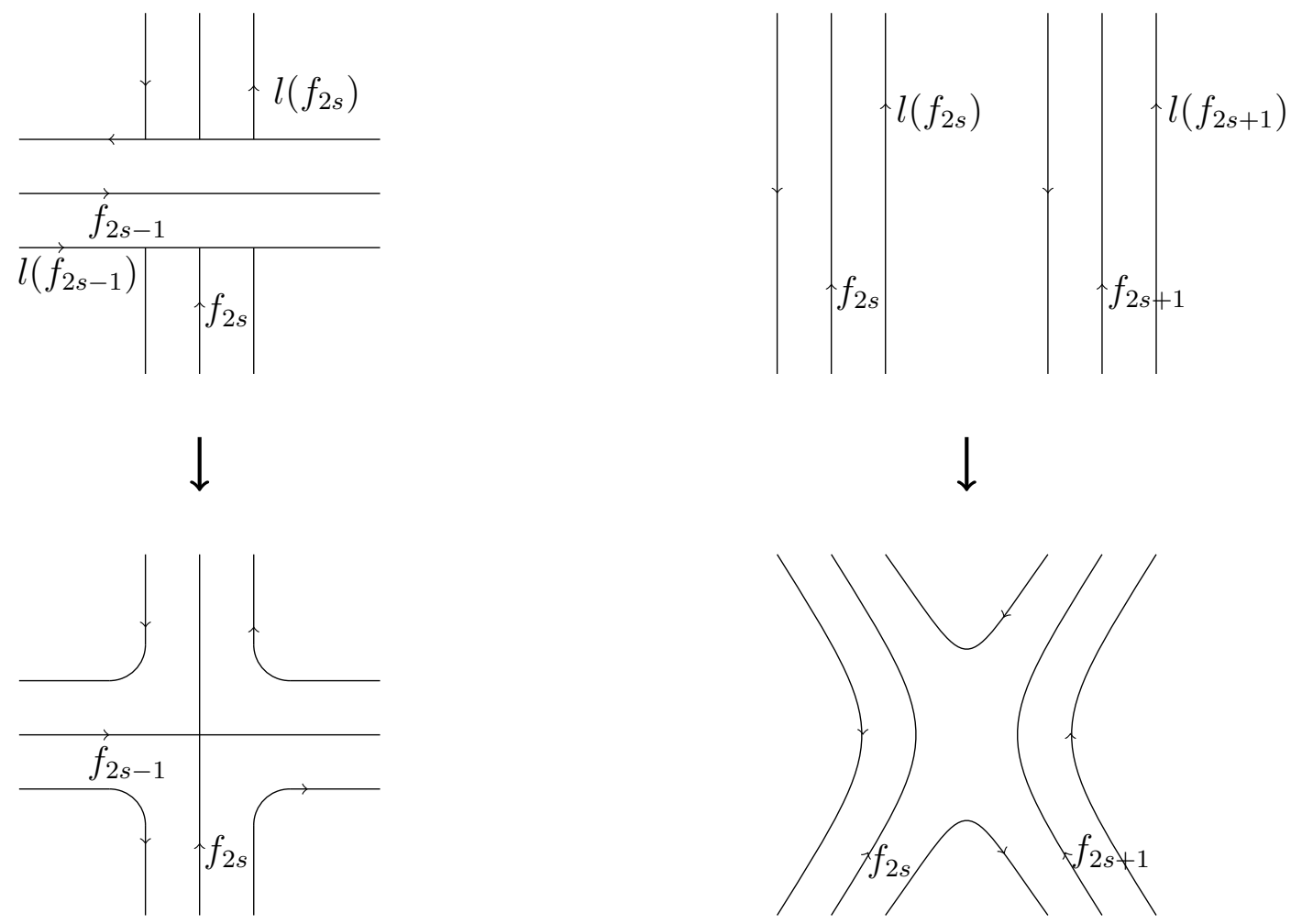

Figure 1: Gluing bands

disjoint simple closed framed curves $f_{i}, 1 \leq i \leq 2 g$, in $M$, such that $l k\left(f_{i}, f_{j}\right)=v_{i j}$ for $j \leq i$. Consider bands around the $f_{i}$, that are images of embeddings $h_{i}:[-1,1] \times S^{1} \hookrightarrow M$ such that $h_{i}\left(\{0\} \times S^{1}\right)=f_{i}$, and $\ell\left(f_{i}\right)=h_{i}\left(\{1\} \times S^{1}\right)$ is the parallel of $f_{i}$ such that $l k\left(f_{i}, \ell\left(f_{i}\right)\right)=v_{i i}$. Connecting these bands as indicated in Figure 1, we get a surface bounded by a knot $K$ which satisfies the required conditions. 
Proof of Proposition 1 1.6. If $V$ is non invertible, there exists $g_{1} \in H_{1}(\Sigma ; \mathbb{Z})$ such that $l k\left(g_{1}, \gamma^{+}\right)=0$ for all $\gamma \in H_{1}(\Sigma ; \mathbb{Z})$. Choose for $g_{1}$ a primitive element of $H_{1}(\Sigma ; \mathbb{Z})$, i.e. such that $g_{1}=k g$ with $k \in \mathbb{Z}$ and $g \in H_{1}(\Sigma ; \mathbb{Z})$ implies $k= \pm 1$. In any symplectic basis of $H_{1}(\Sigma ; \mathbb{Z}), g_{1}$ has coprime coefficients, hence there is $g_{2} \in H_{1}(\Sigma ; \mathbb{Z})$ such that $\left\langle g_{1}, g_{2}\right\rangle_{\Sigma}=1$, where $\langle., .\rangle_{\Sigma}$ denotes the intersection form on $\Sigma$. Consider a symplectic basis $\left(g_{i}\right)_{3 \leq i \leq 2 g}$ of the orthogonal of $\mathbb{Z} g_{1} \oplus \mathbb{Z} g_{2}$ in $H_{1}(\Sigma ; \mathbb{Z})$ with respect to the intersection form. Then $\left(g_{i}\right)_{1 \leq i \leq 2 g}$ is a symplectic basis of $H_{1}(\Sigma ; \mathbb{Z})$, and the associated Seifert matrix $V_{1}$ is a row enlargement of a Seifert matrix $V_{2}$. Since $V$ and $V_{1}$ are associated with the same Seifert surface, they are related by a change of basis of $H_{1}(\Sigma ; \mathbb{Z})$, i.e. they are congruent. Hence $V$ is rationally $\mathrm{S}$-equivalent to the smaller matrix $V_{2}$. Iterating this process, we see that $V$ is rationally S-equivalent to an invertible Seifert matrix $W$, where we consider that there exists an empty matrix, which is invertible. Similarly, $V^{\prime}$ is rationally S-equivalent to an invertible Seifert matrix $W^{\prime}$. The matrices $W$ and $W^{\prime}$ are invertible Seifert matrices that define isomorphic Blanchfield forms.

By Lemma 3.6, there are $\mathbb{Q S K}$-systems $(N, J, S, \underline{\eta}, W)$ and $\left(N^{\prime}, J^{\prime}, S^{\prime}, \underline{\eta^{\prime}}, W^{\prime}\right)$. The rational S-equivalence relation between $V$ and $W$ (resp. $V^{\prime}$ and $W^{\prime}$ ) induces the $\tau$-class of an isomorphism $\zeta: \mathcal{A}(J) \rightarrow \mathcal{A}(K)$ (resp. $\zeta^{\prime}: \mathcal{A}\left(J^{\prime}\right) \rightarrow \mathcal{A}\left(K^{\prime}\right)$ ). By Proposition 3.1, there is an invertible rational symplectic matrix $P$ such that $W^{\prime}=P W P^{t}$ and $P$ induces the $\tau$-class of the isomorphism $\left(\zeta^{\prime}\right)^{-1} \circ \xi \circ \zeta: \mathcal{A}(J) \rightarrow \mathcal{A}\left(J^{\prime}\right)$.

\section{Rational S-equivalence}

In this section, we prove Theorem 1.8 by proving that we can realize a symplectic rational congruence by a finite sequence of enlargements, reductions, and integral symplectic congruences which, for given $\mathbb{Q S K}$-systems, induces the same $\tau$-class of isomorphisms between the Alexander modules as the initial congruence. We first treat a particular type of congruence matrices.

Lemma 4.1. Let $V$ and $W$ be two Seifert matrices such that $\Delta_{n} V \Delta_{n}=W$, where $n$ or $\frac{1}{n}$ is a positive integer, and

$$
\Delta_{n}=\left(\begin{array}{ccccc}
n & & & & \\
& \frac{1}{n} & & 0 & \\
& & 1 & & \\
& 0 & & \ddots & \\
& & & & 1
\end{array}\right)
$$


Then there are enlargements $\tilde{V}$ of $V$ and $\tilde{W}$ of $W$ that are related by an integral symplectic congruence. Furthermore, if $(M, K, \Sigma, \underline{f}, V)$ and $\left(M^{\prime}, K^{\prime}, \Sigma^{\prime}, f^{\prime}, W\right)$ are two $\mathbb{Q} S K$-systems, $W$ can be obtained from $V$ by a sequence of an enlargement, an integral symplectic congruence, and a reduction, which induces the same $\tau$-class of isomorphisms from $\mathcal{A}(K)$ to $\mathcal{A}\left(K^{\prime}\right)$ as the congruence matrix $\Delta_{n}$.

Proof. Assume $n$ is a positive integer.

$$
\begin{aligned}
& \text { Set } V=\left(\begin{array}{ccc}
p & q & \omega^{t} \\
r & s & \rho^{t} \\
\omega & \rho & U
\end{array}\right) \text {. Then } W=\left(\begin{array}{ccc}
n^{2} p & q & n \omega^{t} \\
r & \frac{s}{n^{2}} & \frac{1}{n} \rho^{t} \\
n \omega & \frac{1}{n} \rho & U
\end{array}\right) \text {. Note that } r=q+1 \text {. Set: }
\end{aligned}
$$

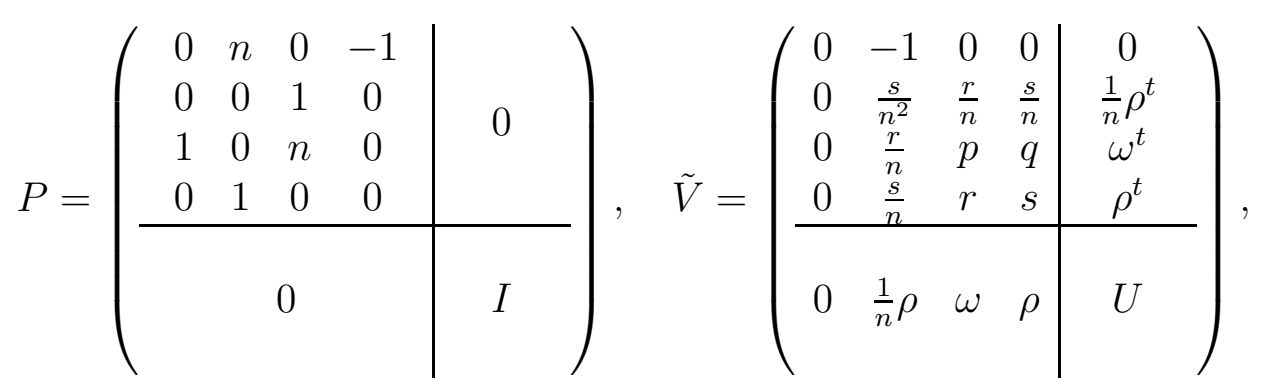

$$
\begin{aligned}
& \text { and } \tilde{W}=\left(\begin{array}{cccc|c}
0 & 0 & 0 & 0 & 0 \\
1 & p & n p & \frac{r}{n} & \omega^{t} \\
0 & n p & n^{2} p & q & n \omega^{t} \\
0 & \frac{r}{n} & r & \frac{s}{n^{2}} & \frac{1}{n} \rho^{t} \\
\hline 0 & \omega & n \omega & \frac{1}{n} \rho & U
\end{array}\right)
\end{aligned}
$$

The matrix $P$ is integral and symplectic, and we have $\tilde{W}=P \tilde{V} P^{t}$.

Let $\left(b_{i}\right)_{1 \leq i \leq 2 g}$ (resp. $\left.\left(b_{i}^{\prime}\right)_{1 \leq i \leq 2 g}\right)$ be a family of generators of $\mathcal{A}(K)$ (resp. $\mathcal{A}\left(K^{\prime}\right)$ ) associated with $V$ (resp. $W$ ). The congruence matrix $\Delta_{n}$ induces the $\tau$-class of the isomorphism $\xi: \mathcal{A}(K) \rightarrow \mathcal{A}\left(K^{\prime}\right)$ such that $\left.\xi\left(b_{i}\right)=\left(\begin{array}{llll}\left(b_{1}^{\prime}\right. & \ldots & b_{2 g}^{\prime}\end{array}\right) \Delta_{n}\right)_{i}$. Let us check that the obtained sequence of an enlargement, an integral symplectic congruence, and a reduction, also induces $\xi$. The matrix $\tilde{V}$ defines a $\mathbb{Q}\left[t^{ \pm 1}\right]$-module $\mathcal{A} \cong \mathcal{A}(K)$ with a generating family $\left(\tilde{b}_{i}\right)_{1 \leq i \leq 2 g+2}$ and relations $\left(\left(\begin{array}{lll}\tilde{b}_{1} & \ldots & \tilde{b}_{2 g+2}\end{array}\right)\left(t \tilde{V}-\tilde{V}^{t}\right)\right)_{j}$. The enlargement of $V$ into $\tilde{V}$ induces the $\tau$-class of the isomorphism $\zeta: \mathcal{A}(K) \rightarrow \mathcal{A}$ such that $\zeta\left(b_{i}\right)=\tilde{b}_{i+2}$. Similarly, define $\mathcal{A}^{\prime},\left(\tilde{b}_{i}^{\prime}\right)_{1 \leq i \leq 2 g+2}$ and $\zeta^{\prime}$. The congruence matrix $P$ induces the $\tau$-class of the isomorphism $\vartheta: \mathcal{A} \rightarrow \mathcal{A}^{\prime}$ such that $\vartheta\left(\tilde{b}_{i}\right)=\left(\left(\begin{array}{lll}\tilde{b}_{1}^{\prime} & \ldots & \tilde{b}_{2 g+2}^{\prime}\end{array}\right) P\right)_{i}$. Set $\xi^{\prime}=\left(\zeta^{\prime}\right)^{-1} \circ \vartheta \circ \zeta$. Let us check that $\xi^{\prime}\left(b_{i}\right)=\xi\left(b_{i}\right)$ for all $i \in\{1, . ., 2 g\}$. For $i \geq 3$, it is obvious. For $i=1$, 
it follows from the relation $\tilde{b}_{2}^{\prime}=0$ given by the first column of the matrix $t \tilde{W}-\tilde{W}^{t}$. For $i=2$, we have $\xi^{\prime}\left(b_{2}\right)=\left(\zeta^{\prime}\right)^{-1}\left(-\tilde{b}_{1}^{\prime}\right)$. Since the second column of $t \tilde{W}-\tilde{W}^{t}$ gives:

$$
\tilde{b}_{1}^{\prime}=(t-1)\left(n p \tilde{b}_{3}^{\prime}+\frac{r}{n} \tilde{b}_{4}^{\prime}+\left(\begin{array}{lll}
\tilde{b}_{5}^{\prime} & \ldots & \tilde{b}_{2 g+2}^{\prime}
\end{array}\right) \omega\right),
$$

and since the first column of $t W-W^{t}$ gives:

$$
b_{2}^{\prime}=-(t-1)\left(n^{2} p b_{1}^{\prime}+r b_{2}^{\prime}+n\left(\begin{array}{lll}
b_{3}^{\prime} & \ldots & b_{2 g}^{\prime}
\end{array}\right) \omega\right),
$$

we have $\xi^{\prime}\left(b_{2}\right)=\frac{1}{n} b_{2}^{\prime}$.

Since $\Delta_{\frac{1}{n}}=\Delta_{n}^{-1}$, the case $\frac{1}{n} \in \mathbb{N} \backslash\{0\}$ follows.

Lemma 4.2. Any symplectic rational matrix $P$ can be written as a product of integral symplectic matrices and matrices $\Delta_{n}$ or $\Delta_{\frac{1}{n}}$ for positive integers $n$.

Proof.

Step 1: There is no loss in assuming that the first column of $P$ is $\left(\begin{array}{c}1 \\ 0 \\ \vdots \\ 0\end{array}\right)$.

Denote by $d$ a common denominator for the terms of the first column of $P$. The matrix $P \Delta_{d}$ has integral coefficients in its first column. Denote by $\delta$ their gcd. The terms of the first column of $P \Delta_{d} \Delta_{\frac{1}{\delta}}$ are coprime integers. There is an integral symplectic matrix $Q$ with the same first column. The matrix $Q^{-1} P \Delta_{d} \Delta_{\frac{1}{\delta}}$ has the required first column.

Step 2: We can assume that the first two columns of $P$ are $\left(\begin{array}{cc}1 & 0 \\ 0 & 1 \\ \vdots & 0 \\ \vdots & \vdots \\ 0 & 0\end{array}\right)$.

The matrix $P^{-1}$ has the same first column as $P$. Since it is symplectic, its second 
column is $\left(\begin{array}{c}x_{1} \\ \vdots \\ x_{2 g}\end{array}\right)$, with $x_{2}=1$. Set:

$$
Q=\left(\begin{array}{ccccccc}
1 & x_{1} & -x_{4} & x_{3} & \ldots & -x_{2 g} & x_{2 g-1} \\
0 & 1 & 0 & \ldots & \ldots & \ldots & 0 \\
0 & x_{3} & 1 & & & & \\
\vdots & \vdots & & \ddots & & 0 & \\
\vdots & \vdots & & & \ddots & & \\
\vdots & \vdots & & 0 & & \ddots & \\
0 & x_{2 g} & & & & & 1
\end{array}\right)
$$

Since $Q$ has the same first two columns as $P^{-1}$, the matrix $P Q$ has the required first two columns. Now, if $n$ is a common denominator for all the $x_{i}$, the matrix $\Delta_{n} Q \Delta_{\frac{1}{n}}$ has integral coefficients, and is symplectic.

Step 3: Induction.

We have $P=\left(\begin{array}{cc}I_{2} & R \\ 0 & Q\end{array}\right)$. Since $P$ is symplectic, $R=0$ and $Q$ is symplectic. Thus we can conclude by induction on $g$.

\section{$5 \quad$ Relating integral Seifert matrices}

In order to prove Theorem 1.10, we want to proceed as for proving Theorem 1.8. Here, we have to avoid enlargements with non integral coefficients. Thus we shall be more careful in the way we decompose rational symplectic congruences. Following Trotter [Tro73], we introduce some formalism. Set $z=(1-t)^{-1}$.

Definition 5.1. A scalar space $\mathcal{A}$ is a finitely generated torsion $\mathbb{Q}\left[t, t^{-1}, z\right]$-module, endowed with a scalar form $[.,]:. \mathcal{A} \times \mathcal{A} \rightarrow \mathbb{Q}$, that is a $\mathbb{Q}$-bilinear non-degenerate antisymmetric form which satisfies:

- $\left[t a_{1}, t a_{2}\right]=\left[a_{1}, a_{2}\right]$,

- $\left[z a_{1}, a_{2}\right]=-\left[a_{1}, t z a_{2}\right]=\left[a_{1},(1-z) a_{2}\right]$,

for all $a_{1}, a_{2} \in \mathcal{A}$. 
Given a $\mathbb{Q S K}$-pair $(M, K)$, define a scalar space structure on the Alexander module $\mathcal{A}(K)$ as follows. Multiplication by $(1-t)$ is an isomorphism of $\mathcal{A}(K)$; define the action of $z$ as its inverse. Define a scalar form on $\mathcal{A}(K)$ by $\left[a_{1}, a_{2}\right]=\chi\left(\phi_{K}\left(a_{1}, a_{2}\right)\right)$ for all $a_{1}, a_{2} \in \mathcal{A}$, where $\chi$ is the map defined before Lemma 3.4.

Definition 5.2. Let $(\mathcal{A},[.,]$.$) be a scalar space of \mathbb{Q}$-dimension $2 g$. A lattice in $\mathcal{A}$ is a free $\mathbb{Z}$-submodule of rank $2 g$. Such a lattice $\Gamma$ is self-dual if, for $a \in \mathcal{A}, a$ is in $\Gamma$ if and only if $[a, x] \in \mathbb{Z}$ for all $x \in \Gamma$. A lattice $\Gamma$ in $\mathcal{A}$ is admissible if it is self-dual and if it satisfies $z \Gamma \subset \Gamma$.

Lemma 5.3. Let $(M, K, \Sigma, f, V)$ be a $\mathbb{Z} S K$-system such that $V$ is invertible over $\mathbb{Q}$. Let $\underline{b}=\left(b_{i}\right)_{1 \leq i \leq 2 g}$ be a basis of $\overline{\mathcal{A}}(K)$ associated with $V$. Let $\Gamma$ be the lattice generated by the basis $\underline{b}$ in $\mathcal{A}(K)$. Then $\Gamma$ is admissible.

Proof. By Lemma 3.5, the matrix of the scalar form on $\mathcal{A}(K)$, with respect to the basis $\underline{b}$, is $-J$. It follows that $\Gamma$ is self-dual. Let $Z$ be the matrix of the action of $z$ in the basis $\underline{b}$. By Corollary $3.3, Z=-V J$. Thus $Z$ has integral coefficients.

The next lemma implies that $\mathcal{A}_{\mathbb{Z}}(K)=\mathbb{Z}\left[t^{ \pm 1}\right] \Gamma \subset \mathcal{A}(K)$, with the notation of Lemma 5.3. Note that multiplication by $(1-t)$ is an isomorphism of $\mathcal{A}_{\mathbb{Z}}(K)$, hence $\mathcal{A}_{\mathbb{Z}}(K)=\Lambda \Gamma$, where $\Lambda=\mathbb{Z}\left[t, t^{-1}, z\right]$.

Lemma 5.4. The integral Alexander module associated with a $\mathbb{Z} S K$-pair has no $\mathbb{Z}$-torsion.

Proof. Let $(M, K, \Sigma, \underline{f}, V)$ be a $\mathbb{Z S K}$-system. Let $\underline{b}=\left(b_{i}\right)_{1 \leq i \leq 2 g}$ be a family of generators of $\mathcal{A}_{\mathbb{Z}}(K)$ associated with $V$. We have:

$$
\mathcal{A}_{\mathbb{Z}}(K)=\frac{\bigoplus_{1 \leq i \leq 2 g} \mathbb{Z}\left[t^{ \pm 1}\right] b_{i}}{\bigoplus_{1 \leq j \leq 2 g} \mathbb{Z}\left[t^{ \pm 1}\right] r_{j}},
$$

where $r_{j}=\sum_{1 \leq i \leq 2 g} W_{i j} b_{i}$ and $W=t V-V^{t}$. Let $p: \underset{1 \leq i \leq 2 g}{\bigoplus} \mathbb{Z}\left[t^{ \pm 1}\right] b_{i} \rightarrow \mathcal{A}_{\mathbb{Z}}(K)$ be the natural projection. Represent the elements of $\underset{1 \leq i \leq 2 g}{\bigoplus} \mathbb{Z}\left[t^{ \pm 1}\right] b_{i}$ by column vectors giving their coordinates in the basis $\underline{b}$.

Let $A \in \underset{1 \leq i \leq 2 g}{\bigoplus} \mathbb{Z}\left[t^{ \pm 1}\right] b_{i}$. Assume $k A \in \operatorname{ker}(p)$ for a non trivial integer $k$. Then there is $X \in \underset{1 \leq i \leq 2 g}{\bigoplus} \mathbb{Z}\left[t^{ \pm 1}\right] b_{i}$ such that $k A=W X$. Thus:

$$
k \operatorname{Cof}(W) A=\operatorname{det}(W) X=\Delta(t) X,
$$


where $\operatorname{Cof}(W)$ is the cofactor matrix of $W$ and $\Delta(t)$ is the Alexander polynomial of $(M, K)$. Hence $k$ divides each coefficient of $\Delta(t) X$. Since $\Delta(1)=1$, it implies that $X=k Y$ for some $Y \in \bigoplus_{1 \leq i \leq 2 g} \mathbb{Z}\left[t^{ \pm 1}\right] b_{i}$. Thus $A=W Y \in \operatorname{ker}(p)$.

Let $\Gamma$ be a lattice in a scalar space $\mathcal{A}$. A basis $\underline{b}=\left(b_{i}\right)_{1 \leq i \leq 2 g}$ of $\Gamma$ is symplectic if the matrix of the scalar form with respect to $\underline{b}$ is $-J$.

Lemma 5.5. Let $(\mathcal{A},[.,]$.$) be a scalar space. Let \Gamma$ be a lattice in $\mathcal{A}$. Then $\Gamma$ is self-dual if and only if it has a symplectic basis.

Proof. Assume $\Gamma$ is self-dual. Let $\underline{b}=\left(b_{i}\right)_{1 \leq i \leq 2 g}$ be any basis of $\Gamma$. Set $s_{i}=\left[b_{1}, b_{i}\right]$. The self-duality condition implies that the non trivial $s_{i}$ are coprime. Hence there are integers $u_{i}$ such that $\sum_{i=1}^{2 g} u_{i} s_{i}=1$. Set $b_{2}^{\prime}=\sum_{i=1}^{2 g} u_{i} b_{i}$, so that $\left[b_{1}, b_{2}^{\prime}\right]=1$. Let $\mathcal{B}$ be the orthogonal in $\mathcal{A}$ of $\mathbb{Q} b_{1} \oplus \mathbb{Q} b_{2}^{\prime}$ with respect to the scalar form. For $x \in \Gamma, y=x-\left[x, b_{2}^{\prime}\right] b_{1}-\left[b_{1}, x\right] b_{2}^{\prime} \in \Gamma$ is orthogonal to $b_{1}$ and $b_{2}^{\prime}$. It follows that $\Gamma$ is the direct sum, orthogonal with respect to the scalar form, of $\mathbb{Z} b_{1} \oplus \mathbb{Z} b_{2}^{\prime}$ and $\mathcal{B} \cap \Gamma$. Conclude by induction on $g$ that $\Gamma$ has a symplectic basis. The reverse implication is easy.

Lemma 5.6. Let $(\mathcal{A},[.,]$.$) be a scalar space of \mathbb{Q}$-dimension $2 g$. Let $\Gamma$ be an admissible lattice in $\mathcal{A}$. Let $\underline{b}$ be a symplectic basis of $\Gamma$. Let $Z$ be the matrix of the action of $z$ in the basis $\underline{b}$. Set $V=Z J$. Then $V$ is an integral Seifert matrix.

Proof. By definition of a scalar form, we have $Z^{t} J=J(I-Z)$. It follows that $V-V^{t}=J$. Integrality follows from the admissibility condition.

The matrix $V$ defined in the above lemma is the Seifert matrix associated with $\Gamma$ and $\underline{b}$.

Definition 5.7. Let $(\mathcal{A},[.,]$.$) be a scalar space of \mathbb{Q}$-dimension $2 g$. For $n \in \mathbb{N} \backslash\{0\}$, two lattices $\Gamma$ and $\Gamma^{\prime}$ in $\mathcal{A}$ are $n$-adjacent if $\frac{\Gamma}{\Gamma \cap \Gamma^{\prime}} \cong \frac{\mathbb{Z}}{n \mathbb{Z}}$ and $\frac{\Gamma^{\prime}}{\Gamma \cap \Gamma^{\prime}} \cong \frac{\mathbb{Z}}{n \mathbb{Z}}$. Two lattices $\Gamma$ and $\Gamma^{\prime}$ are adjacent if they are $n$-adjacent for some $n \in \mathbb{N} \backslash\{0\}$.

The following proposition is the object of Section 3 in [Tro73], althought it is not stated like this.

Proposition 5.8 (Trotter). Let $(\mathcal{A},[.,]$.$) be a scalar space. Let \Gamma$ and $\Gamma^{\prime}$ be admissible lattices in $\mathcal{A}$ such that $\Lambda \Gamma=\Lambda \Gamma^{\prime}$. Then there is a sequence of admissible lattices $\Gamma_{0}=$ $\Gamma, \Gamma_{1}, \ldots, \Gamma_{k}=\Gamma^{\prime}$, such that, for $1 \leq i \leq k, \Gamma_{i-1}$ and $\Gamma_{i}$ are adjacent, and $z \Gamma_{i-1} \subset \Gamma_{i}$ or $z \Gamma_{i} \subset \Gamma_{i-1}$. 
This result implies that two integral Seifert matrices, invertible over $\mathbb{Q}$, which define the same integral Alexander module, can be related by integral congruences, and congruences with congruence matrices $\Delta_{n}$ (see Lemma 4.1 for the definition of $\Delta_{n}$ ). The proposition says more, with the last condition on the lattices, and we will use this to prove that these $\Delta_{n}$-congruences can be realized by integral S-equivalences. We first check that we can always use symplectic bases of the lattices.

An element $a$ of a lattice $\Gamma$ is primitive if the equality $a=k b$ with $k \in \mathbb{Z}$ and $b \in \Gamma$ implies $k= \pm 1$. It is easy to see that it is necessary and sufficient for $a$ to be primitive that the non trivial coefficients of $a$ in any basis of $\Gamma$ are coprime. It is also easy to see that, if $\Gamma$ is a self-dual lattice in a scalar space $(\mathcal{A},[.,]$.$) , then a \in \Gamma$ is primitive if and only if there is $x \in \Gamma$ such that $[a, x]=1$.

Lemma 5.9. Let $(\mathcal{A},[.,]$.$) be a scalar space. Let \Gamma$ and $\Gamma^{\prime}$ be $n$-adjacent lattices in $\mathcal{A}$. Assume $\Gamma$ and $\Gamma^{\prime}$ are self-dual. Then there is a symplectic basis $\left(b_{i}\right)_{1 \leq i \leq 2 g}$ of $\Gamma$ such that $\left(n b_{1}, \frac{1}{n} b_{2}, b_{3}, \ldots, b_{2 g}\right)$ is a symplectic basis of $\Gamma^{\prime}$.

Proof. Let $g$ be an element of $\Gamma$ which generates $\frac{\Gamma}{\Gamma \cap \Gamma^{\prime}} \cong \frac{\mathbb{Z}}{n \mathbb{Z}}$. Let $b_{1}$ be a generator of $(\mathbb{Q} g) \cap \Gamma \cong \mathbb{Z}$. Note that $b_{1}$ also generates $\frac{\Gamma}{\Gamma \cap \Gamma^{\prime}}$. Let us prove that $n b_{1}$ is a primitive element of $\Gamma^{\prime}$. Assume $n b_{1}=k \gamma$ for some $k \in \mathbb{Z}$ and $\gamma \in \Gamma^{\prime}$. For any proper divisor $n^{\prime}$ of $n, n^{\prime} b_{1}$ is not in $\Gamma^{\prime}$. Hence $n$ and $k$ are coprime. Since $n \gamma \in \Gamma$ and $k \gamma \in \Gamma$, it implies that $\gamma \in \Gamma$. Thus $\gamma \in \mathbb{Z}\left(n b_{1}\right)$, and $k= \pm 1$. Hence $n b_{1}$ is primitive in $\Gamma^{\prime}$, and there is $b_{2}^{\prime} \in \Gamma^{\prime}$ such that $\left[n b_{1}, b_{2}^{\prime}\right]=1$. Set $b_{2}=n b_{2}^{\prime}$. Let $\mathcal{B}$ be the orthogonal of $\mathbb{Q} b_{1} \oplus \mathbb{Q} b_{2}$ in $\mathcal{A}$ with respect to the scalar form. Check that $\Gamma=\left(\mathbb{Z} b_{1} \oplus \mathbb{Z} b_{2}\right) \oplus^{\perp}(\mathcal{B} \cap \Gamma)$ and $\Gamma^{\prime}=\left(\mathbb{Z} n b_{1} \oplus \mathbb{Z} \frac{1}{n} b_{2}\right) \oplus^{\perp}\left(\mathcal{B} \cap \Gamma^{\prime}\right)$. Thus $\mathcal{B} \cap \Gamma=\mathcal{B} \cap \Gamma^{\prime}$ is a self-dual lattice in $\mathcal{B}$. Let $\left(b_{3}, \ldots, b_{2 g}\right)$ be a symplectic basis of this lattice. Then the basis $\left(b_{i}\right)_{1 \leq i \leq 2 g}$ of $\Gamma$ satisfies the required conditions.

Lemma 5.10. Let $(\mathcal{A},[.,]$.$) be a scalar space. Let \Gamma$ and $\Gamma^{\prime}$ be $n$-adjacent admissible lattices in $\mathcal{A}$. Let $\underline{b}=\left(b_{i}\right)_{1 \leq i \leq 2 g}$ be a symplectic basis of $\Gamma$ such that $\underline{b}^{\prime}=\left(\frac{1}{n} b_{1}, n b_{2}, b_{3}, \ldots, b_{2 g}\right)$ is a symplectic basis of $\Gamma^{\prime}$. Let $V$ and $V^{\prime}$ be the Seifert matrices associated with $\underline{b}$ and $\underline{b}^{\prime}$ respectively. Then $V^{\prime}=\Delta_{n} V \Delta_{n}$. If $z \Gamma \subset \Gamma^{\prime}$, then $V^{\prime}$ can be obtained from $V$ by an integral S-equivalence which induces the same $\tau$-class of isomorphisms as the congruence $V^{\prime}=\Delta_{n} V \Delta_{n}$.

Proof. Let $Z$ (resp. $Z^{\prime}$ ) be the matrix of the action of $z$ in the basis $\underline{b}$ (resp. $\underline{b}^{\prime}$ ). Note that $\Delta_{n} Z=Z^{\prime} \Delta_{n}$. By Corollary 3.3, $V=Z J$ and $V^{\prime}=Z^{\prime} J$. It follows that $V^{\prime}=\Delta_{n} V \Delta_{n}$.

To prove the second statement, it suffices to prove that we can proceed as in Lemma 4.1, i.e. that the coefficient $V_{21}$ of $V$ is divisible by $n$. Since $z \Gamma \subset\left(\Gamma \cap \Gamma^{\prime}\right)$, each $z b_{i}$ is a 
linear combination of $b_{1}, n b_{2}, b_{3}, \ldots, b_{2 g}$. It follows that $Z$ has its second row divisible by $n$. Hence $V=Z J$ also has its second row divisible by $n$.

Proposition 5.11. Let $(M, K, \Sigma, \underline{f}, V)$ and $\left(M^{\prime}, K^{\prime}, \Sigma^{\prime}, \underline{f}^{\prime}, V^{\prime}\right)$ be two $\mathbb{Z} S K$-systems. Let $\xi: \mathcal{A}_{\mathbb{Z}}(K) \rightarrow \mathcal{A}_{\mathbb{Z}}\left(K^{\prime}\right)$ be an isomorphism which preserves the Blanchfield form. Assume $V$ and $V^{\prime}$ are invertible over $\mathbb{Q}$. Then $V$ and $V^{\prime}$ are related by an integral $S$-equivalence which canonically induces the $\tau$-class of $\xi$.

Proof. Let $\underline{b}=\left(b_{i}\right)_{1 \leq i \leq 2 g}$ and $\underline{b}^{\prime}=\left(b_{i}^{\prime}\right)_{1 \leq i \leq 2 g}$ be bases of $\mathcal{A}(K)$ and $\mathcal{A}\left(K^{\prime}\right)$ respectively, associated with $V$ and $V^{\prime}$. Let $P$ be the matrix of $\xi \otimes_{\mathbb{Z}} I d_{\mathbb{Q}}$ with respect to the bases $\underline{b}$ and $\underline{b}^{\prime}$. By Proposition [3.1, $V^{\prime}=P V P^{t}$. Let $\Gamma$ (resp. $\Gamma^{\prime}$ ) be the lattice in $\mathcal{A}\left(K^{\prime}\right)$ generated by the $\xi\left(b_{i}\right)$ (resp. by the $b_{i}^{\prime}$ ). By Lemma 5.3, $\Gamma$ and $\Gamma^{\prime}$ are admissible. It is clear that $\Lambda \Gamma=\Lambda \Gamma^{\prime}$. Hence, by Proposition 5.8, there is a sequence of admissible lattices $\Gamma_{0}=\Gamma, \Gamma_{1}, \ldots, \Gamma_{k}=\Gamma^{\prime}$ in $\mathcal{A}\left(K^{\prime}\right)$, such that, for $1 \leq j \leq k, \Gamma_{j-1}$ and $\Gamma_{j}$ are adjacent, and $z \Gamma_{j} \subset \Gamma_{j-1}$ or $z \Gamma_{j-1} \subset \Gamma_{j}$.

By Lemma 5.9. for $1 \leq j \leq k$, there are Seifert matrices $V_{j}$ and $\hat{V}_{j-1}$ associated with $\Gamma_{j}$ and $\Gamma_{j-1}$ respectively, and with symplectic bases of these lattices, such that $V_{j}=\Delta_{n_{j}} \hat{V}_{j-1} \Delta_{n_{j}}$, where $n_{j}$ is an integer or the inverse of an integer. Set $V_{0}=V$ and $\hat{V}_{k}=V^{\prime}$. For $0 \leq j \leq k$, the matrices $V_{j}$ and $\hat{V}_{j}$ are Seifert matrices associated with symplectic bases of the same lattice, and the change of basis provides an integral symplectic matrix $P_{j}$ such that $\hat{V}_{j}=P_{j} V_{j} P_{j}^{t}$. We have $P=P_{k} \Delta_{n_{k}} P_{k-1} \ldots \Delta_{n_{1}} P_{0}$, and the $\tau$-class of the composition of the successive isomorphisms induced by the successive congruences is the $\tau$-class of the isomorphism induced by the congruence $V^{\prime}=P V P^{t}$. Conclude with Lemma 5.10 .

Proof of Theorem 1.10. Proceed as in the proof of Proposition 1.6.

\section{Topological realization of matrix relations}

In this section, we prove Theorem 1.13 and Theorem 1.14

Lemma 6.1. Let $M$ be a $\mathbb{Q} H S$ (resp. $\mathbb{Z} H S$ ). Let $\Sigma$ be a genus g closed connected surface embedded in $M$. Then $M \backslash \Sigma$ has exactly two connected components, whose closures are $\mathbb{Q} H H^{\prime} s$ (resp. $\mathbb{Z} H H^{\prime}$ 's) of genus $g$.

Proof. Any point of $M \backslash \Sigma$ can be connected to a point of $\Sigma \times[-1,1]$ in $M \backslash \Sigma$. Since $(\Sigma \times[-1,1]) \backslash \Sigma$ has two connected components, $M \backslash \Sigma$ has at most two connected components. Let $x_{1}$ and $x_{2}$ be points of $(\Sigma \times[-1,1]) \backslash \Sigma$, one in each connected component. 
If there were a path from $x_{1}$ to $x_{2}$ in $M \backslash \Sigma$, we could construct a closed curve in $M$ which would meet $\Sigma$ exactly once. Since $M$ is a $\mathbb{Q H S}$, this is not possible. Hence $M \backslash \Sigma$ has exactly two connected components. Let $A_{1}$ and $A_{2}$ be their closures. Note that $\partial A_{1}=\partial A_{2}=\Sigma($ up to orientation).

For $i=1,2$, we have $H_{3}\left(A_{i} ; \mathbb{Z}\right)=0$ and $H_{0}\left(A_{i} ; \mathbb{Z}\right)=\mathbb{Z}$. The Mayer-Vietoris sequence associated with $M=A_{1} \cup A_{2}$ yields the exact sequence:

$$
H_{3}(M ; \mathbb{Z}) \stackrel{\partial}{\longrightarrow} H_{2}(\Sigma ; \mathbb{Z}) \longrightarrow H_{2}\left(A_{1} ; \mathbb{Z}\right) \oplus H_{2}\left(A_{2} ; \mathbb{Z}\right) \longrightarrow 0
$$

The map $\partial$ is an isomorphism that identifies the fundamental classes. Thus $H_{2}\left(A_{1} ; \mathbb{Z}\right)=$ $H_{2}\left(A_{2} ; \mathbb{Z}\right)=0$. It follows that $A_{1}$ and $A_{2}$ are $\mathbb{Q H H}$ 's. Their genus is given by their boundary.

Assume $M$ is a $\mathbb{Z H S}$. The Mayer-Vietoris sequence associated with $M=A_{1} \cup A_{2}$ yields an isomorphism $H_{1}(\Sigma ; \mathbb{Z}) \cong H_{1}\left(A_{1} ; \mathbb{Z}\right) \oplus H_{1}\left(A_{2} ; \mathbb{Z}\right)$. Hence, for $i=1,2, H_{1}\left(A_{i} ; \mathbb{Z}\right)$ is torsion-free, thus $A_{i}$ is a $\mathbb{Z H H}$.

Lemma 6.2. Let $(M, K, \Sigma, f, V)$ and $\left(M^{\prime}, K^{\prime}, \Sigma^{\prime}, f^{\prime}, V^{\prime}\right)$ be $\mathbb{Q} S K$-systems. Let $P$ be an integral symplectic matrix. Assume $V^{\prime}=P V P^{t}$. Then there is a null LP-surgery $\left(\frac{B}{A}\right)$ in $M \backslash K$ such that $\left(M^{\prime}, K^{\prime}\right) \cong(M, K)\left(\frac{B}{A}\right)$ and the $\tau$-class of the isomorphism $\xi: \mathcal{A}(K) \rightarrow$ $\mathcal{A}\left(K^{\prime}\right)$ induced by this surgery is the $\tau$-class induced by $P$.

Proof. There is a homeomorphism $h: \Sigma \rightarrow \Sigma^{\prime}$, such that the matrix of the induced isomorphism $h_{*}: H_{1}(\Sigma ; \mathbb{Z}) \rightarrow H_{1}\left(\Sigma^{\prime} ; \mathbb{Z}\right)$ with respect to the bases $\left(f_{i}\right)_{1 \leq i \leq 2 g}$ and $\left(f_{i}^{\prime}\right)_{1 \leq i \leq 2 g}$ is $\left(P^{t}\right)^{-1}$ (see [FM12, theorem 6.4]). Let $\hat{\Sigma}$ be obtained from $\Sigma$ by adding a band glued along $\partial \Sigma$, so that $\hat{\Sigma}$ is homeomorphic to $\Sigma$, and contains $\Sigma$ and $K$ in its interior. Let $H=\hat{\Sigma} \times[-1,1]$ be a regular neighborhood of $\Sigma$. Similarly, define $\hat{\Sigma}^{\prime}$ and $H^{\prime}$. Extend $h$ to a homeomorphism $h: \hat{\Sigma} \rightarrow \hat{\Sigma}^{\prime}$, and then extend it by product with the identity to a homeomorphism $h: H \rightarrow H^{\prime}$.

Let $A=M \backslash \operatorname{Int}(H)$ and let $B=M^{\prime} \backslash \operatorname{Int}\left(H^{\prime}\right)$. By Lemma 6.1, $A$ (resp. $B$ ) is a $\mathbb{Q} H H$, and it is clearly null in $M \backslash K$ (resp. $\left.M^{\prime} \backslash K^{\prime}\right)$. The $\mathbb{Q S K}$-pair $\left(M^{\prime}, K^{\prime}\right)$ is obtained from $(M, K)$ by the surgery $\left(\frac{B}{A}\right)$. Let us prove that the homeomorphism $h_{\mid \partial A}: \partial A \rightarrow \partial B$ preserves the Lagrangian.

For $1 \leq i \leq 2 g$, let $e_{i} \subset \partial H$ be a meridian of $f_{i}$. The Lagrangian $\mathcal{L}_{A}$ is generated by the $\alpha_{i}=f_{i}^{+}-\sum_{1<j<2 q} V_{j i} e_{j}$, where $f_{i}^{+}$is the copy of $f_{i}$ in $(\Sigma \times\{1\}) \subset H$. Similarly, define the $e_{i}^{\prime},\left(f_{i}^{\prime}\right)^{+}$and $\alpha_{i}^{\prime}$. Since $h: H \rightarrow H^{\prime}$ is a homeomorphism, $\left(h_{\mid \partial H}\right)_{*}\left(\mathcal{L}_{H}\right)=\mathcal{L}_{H^{\prime}}$. Hence $\left(h_{\mid \partial H}\right)_{*}\left(e_{i}\right)$ is a linear combination of the $e_{j}^{\prime}$. Since $\left\langle h\left(e_{i}\right), h\left(f_{j}^{+}\right)\right\rangle_{\partial H^{\prime}}=\left\langle e_{i}^{\prime},\left(f_{j}^{\prime}\right)^{+}\right\rangle_{\partial H^{\prime}}=\delta_{i j}$, an easy computation gives $\left(h_{\mid \partial H}\right)_{*}\left(e_{i}\right)=\left(\left(\begin{array}{lll}e_{1}^{\prime} & \ldots & e_{2 g}^{\prime}\end{array}\right) P\right)_{i}$. It follows that $\left(h_{\mid \partial H}\right)_{*}\left(\alpha_{i}\right)=$

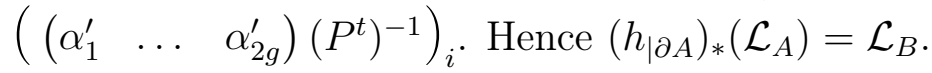


The relation between the $h\left(e_{i}\right)$ and the $e_{i}^{\prime}$ shows that the isomorphism $\xi$ induced by the surgery is in the $\tau$-class of isomorphisms induced by the congruence matrix $P$.

The previous proof still works when $\mathbb{Q}$ is replaced by $\mathbb{Z}$. Therefore:

Lemma 6.3. Let $(M, K, \Sigma, f, V)$ and $\left(M^{\prime}, K^{\prime}, \Sigma^{\prime}, f^{\prime}, V^{\prime}\right)$ be $\mathbb{Z} S K$-systems. Let $P$ be an integral symplectic matrix. Assume $V^{\prime}=P V P^{t}$. Then there is an integral null LPsurgery $\left(\frac{B}{A}\right)$ in $M \backslash K$ such that $\left(M^{\prime}, K^{\prime}\right) \cong(M, K)\left(\frac{B}{A}\right)$ and the $\tau$-class of the isomorphism $\xi: \mathcal{A}_{\mathbb{Z}}(K) \rightarrow \mathcal{A}_{\mathbb{Z}}\left(K^{\prime}\right)$ induced by this surgery is the $\tau$-class induced by $P$.

Lemma 6.4. Let $(M, K, \Sigma, f, V)$ be a $\mathbb{Q} S K$-system. Let $W$ be an enlargement of $V$. Then there is a $\mathbb{Q} S K$-system $\left(M^{\prime}, \bar{K}^{\prime}, \Sigma^{\prime}, f^{\prime}, W\right)$ such that $\left(M^{\prime}, K^{\prime}\right)$ can be obtained from $(M, K)$ by a single null LP-surgery, and the surgery and the enlargement induce the same $\tau$-class of isomorphisms from $\mathcal{A}(K)$ to $\mathcal{A}\left(K^{\prime}\right)$.

Proof. We have

$$
W=\left(\begin{array}{ccc}
0 & 0 & 0 \\
1 & x & \rho^{t} \\
0 & \rho & V
\end{array}\right) \text { or }\left(\begin{array}{ccc}
0 & -1 & 0 \\
0 & x & \rho^{t} \\
0 & \rho & V
\end{array}\right)
$$

We want to add a tube to $\Sigma$, whose linking numbers with the $f_{i}$ are given by $\rho$. This may not be possible in $M$, so we first modify $M$ by null LP-surgeries.

Set $\rho=\left(\begin{array}{c}\frac{c_{1}}{d_{1}} \\ \vdots \\ \frac{c_{2 g}}{d_{2 g}}\end{array}\right)$, where the $c_{i}$ and $d_{i}$ are integers, and $d_{i}>0$. Consider trivial knots $J_{i}$, disjoint from $\Sigma$, such that $l k\left(J_{i}, f_{j}\right)=\delta_{i j} c_{i}$. For each $i$, consider a tubular neighborhood $T\left(J_{i}\right)$ of $J_{i}$. By [Mou12a, Lemma 2.5], there are rational homology tori $A_{i}$ that satisfy:

- $H_{1}\left(\partial A_{i} ; \mathbb{Z}\right)=\mathbb{Z} \alpha_{i} \oplus \mathbb{Z} \beta_{i}$, with $<\alpha_{i}, \beta_{i}>=1$,

- $\beta_{i}=d_{i} \gamma_{i}$ in $H_{1}\left(A_{i} ; \mathbb{Z}\right)$, where $\gamma_{i}$ is a curve in $A_{i}$,

- $H_{1}\left(A_{i} ; \mathbb{Z}\right)=\mathbb{Z} \gamma_{i} \oplus \frac{\mathbb{Z}}{d_{i} \mathbb{Z}} \alpha_{i}$.

Let $N$ be the manifold obtained from $M$ by the null LP-surgeries $\left(\frac{A_{i}}{T\left(J_{i}\right)}\right)$, where the identifications $\partial T\left(J_{i}\right)=\partial A_{i}$ identify $\alpha_{i}$ with a meridian of $J_{i}$, and $\beta_{i}$ with a parallel of $J_{i}$ that does not link $J_{i}$. We get $l k\left(\gamma_{i}, f_{j}\right)=\delta_{i j} \frac{c_{i}}{d_{i}}$.

In $N$, consider a ball $B$ disjoint from $\Sigma$ and all the $A_{i}$. Consider a rational homology ball $B^{\prime}$ that contains a curve $\gamma_{0}$ with self-linking $\left(x-\sum_{1 \leq i, j \leq 2 g} l k\left(\gamma_{i}, \gamma_{j}\right)\right) \bmod \mathbb{Z}$. Set $M^{\prime}=N\left(\frac{B^{\prime}}{B}\right)$, and $K^{\prime}=K$. 

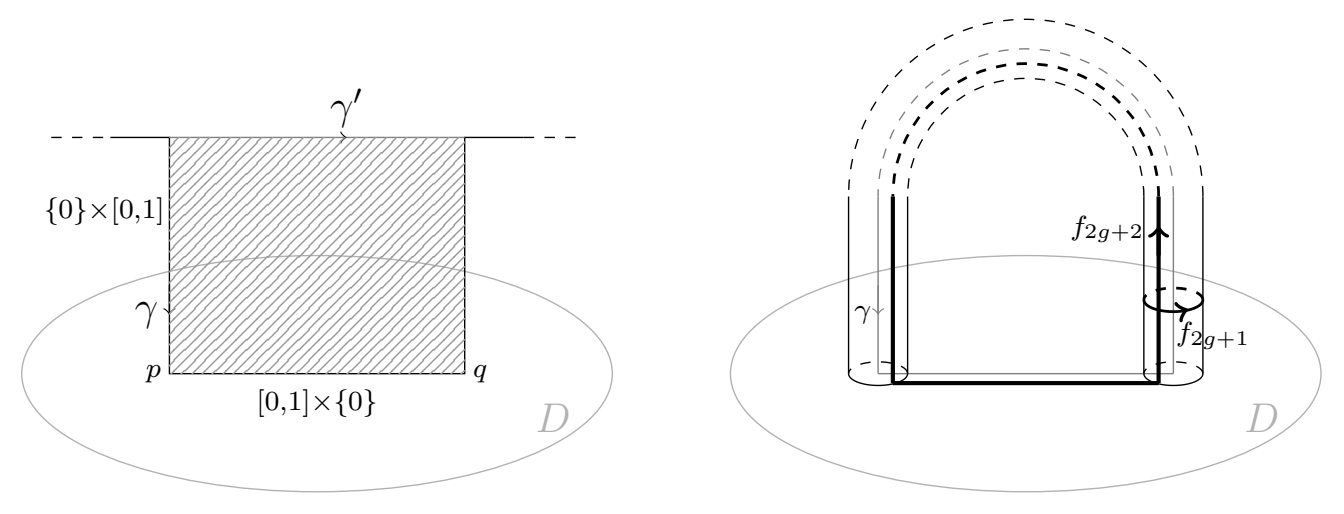

Figure 2: Adding a tube to $\Sigma$

Define a curve $\gamma^{\prime}$ in $M^{\prime}$ as a band sum of the $\gamma_{i}$ for $0 \leq i \leq 2 g$, with bands outside $\Sigma$. Consider a disk $D$ in $\Sigma$, and two distinct points $p$ and $q$ in $D$. Consider an embedded band $[0,1] \times[0,1]$ in $M^{\prime}$ such that $[0,1] \times\{0\}=([0,1] \times[0,1]) \cap \Sigma$ is a curve from $p$ to $q$ in $D,[0,1] \times\{1\}=([0,1] \times[0,1]) \cap \gamma^{\prime}$, and the tangent vector to $\{0\} \times[0,1]$ at $\{0\} \times\{0\}$ is the positive normal vector of $\Sigma$ if $W$ is a row enlargement of $V$, and the negative one if it is a column enlargement. Figure 2 represents the first case. Now set $\gamma=\left(\gamma^{\prime} \cup \partial([0,1] \times\right.$ $[0,1])) \backslash(] 0,1[\times\{1\})$, and construct a surface $\Sigma^{\prime}$ by adding a tube around $\gamma \backslash([0,1] \times\{0\})$ to $\Sigma$. The surface $\Sigma^{\prime}$ is a Seifert surface for $K^{\prime}$. On $\Sigma^{\prime}$, consider a meridian $f_{2 g+1}$ of the tube and a parallel $f_{2 g+2}$ of $\gamma$ such that $<f_{2 g+1}, f_{2 g+2}>_{\Sigma^{\prime}}=1$ and $l k\left(f_{2 g+2}, \gamma\right)=x$. Note that the orientation of the meridian depends on the type of enlargement. The Seifert matrix associated with $\Sigma^{\prime}$ with respect to the basis $\left(f_{2 g+1}, f_{2 g+2}, f_{1}, \ldots, f_{2 g}\right)$ is $W$.

Since the different $\mathbb{Q} H H$ 's replaced by surgery are disjoint, they can be connected by tubes. Thus $\left(M^{\prime}, K^{\prime}\right)$ can be obtained from $(M, K)$ by one surgery on a genus $2 g \mathbb{Q H H}$. Let $\left(b_{i}\right)_{1 \leq i \leq 2 g}$ (resp. $\left(b_{i}^{\prime}\right)_{1 \leq i \leq 2 g+2}$ ) be a family of generators of $\mathcal{A}(K)$ (resp. $\mathcal{A}\left(K^{\prime}\right)$ ) associated with $V$ (resp. $W$ ). The $b_{i}^{\prime}$ can be chosen so that the isomorphism $\xi: \mathcal{A}(K) \rightarrow \mathcal{A}\left(K^{\prime}\right)$ induced by the surgery satisfies $\xi\left(b_{i}\right)=b_{i+2}^{\prime}$.

Lemma 6.5. Let $(M, K, \Sigma, \underline{f}, V)$ be a $\mathbb{Z} S K$-system. Let $W$ be an integral enlargement of $V$. Then there is a $\mathbb{Z} S K$-system $\left(M, K, \Sigma^{\prime}, \underline{f}^{\prime}, W\right)$ and the enlargement induces the $\tau$-class of the identity of $\mathcal{A}_{\mathbb{Z}}(K)$.

Proof. In the previous proof, replace $\mathbb{Q}$ by $\mathbb{Z}$, and remove the definition of the surgery, since any integral linking can be realised in any $\mathbb{Z H S}$.

Proof of Theorem 1.13. Let $V$ and $V^{\prime}$ be Seifert matrices associated with $(M, K)$ and $\left(M^{\prime}, K^{\prime}\right)$ respectively. By Theorem 1.8, $V^{\prime}$ can be obtained from $V$ by a sequence of 
enlargements, reductions, and integral symplectic congruences which induces the $\tau$-class of the isomorphism $\xi$. This provides a finite sequence $V_{1}, V_{2}, . ., V_{n}$ of Seifert matrices such that $V_{1}=V, V_{n}=V^{\prime}$, and $V_{i+1}$ is obtained from $V_{i}$ by one of these equivalences. By Lemma 3.6, for each $i$, we can fix a $\mathbb{Q S K}$-system $\mathcal{S}_{i}$ with Seifert matrix $V_{i}$. To see that $\mathcal{S}_{i+1}$ can be obtained from $\mathcal{S}_{i}$ by one, or two successive, null LP-surgeries, which induce the required $\tau$-class of isomorphisms, apply Lemma 6.2 in the case of a (possibly trivial) congruence, and apply Lemma 6.4 in the case of an enlargement or a reduction.

Similarly, Theorem 1.14 can be deduced from Theorem 1.10, and Lemmas [3.6, 6.3, and 6.5 .

\section{Sequences of LP-surgeries}

In this section, we prove Proposition 1.15.

Lemma 7.1. There exist two knots in $S^{3}$ which have isomorphic rational Blanchfield forms, and different integral Alexander modules.

Proof. In $S^{3}$, consider a knot $K$ with Seifert matrix $\left(\begin{array}{cc}-1 & 0 \\ 1 & 2\end{array}\right)$, and a knot $K^{\prime}$ with Seifert matrix $\left(\begin{array}{ll}3 & 1 \\ 2 & 0\end{array}\right)$. Their Alexander modules have presentation matrices $\left(\begin{array}{cc}1-t & -1 \\ t & 2 t-2\end{array}\right)$ and $\left(\begin{array}{cc}3 t-3 & t-2 \\ 2 t-1 & 0\end{array}\right)$. Both have Alexander polynomial $\Delta(t)=(2 t-1)(2-t)$. Since it is the product of two dual non symmetric prime polynomials, their rational Blanchfield forms are isomorphic (see [Mou12b, Lemma 3.6]). But $K$ has integral Alexander module $\frac{\mathbb{Z}\left[t^{ \pm 1}\right]}{(\Delta(t))}$, whereas the integral Alexander module of $K^{\prime}$ has a non trivial second elementary ideal (the $k$-th elementary ideal associated with a $\mathbb{Z}\left[t^{ \pm 1}\right]$-module is the ideal of $\mathbb{Z}\left[t^{ \pm 1}\right]$ generated by the minors of size $n-k+1$ of a presentation matrix with $n$ generators of the module, see [Lic97, Chapter 6]). Indeed, this ideal is generated by $(t-2)$ and $(2 t-1)$ in $\mathbb{Z}\left[t^{ \pm 1}\right]$, so the evaluation at $t=-1$ maps it onto $3 \mathbb{Z}$.

Proof of Proposition 1.15. Consider the $\mathbb{Q}$ SK-pairs $\left(S^{3}, K\right)$ and $\left(S^{3}, K^{\prime}\right)$ of Lemma 7.1 . By Theorem 1.13, $\left(S^{3}, K^{\prime}\right)$ can be obtained from $\left(S^{3}, K\right)$ by a finite sequence of null LP-surgeries. Suppose we can restrict to a single surgery $\left(\frac{B}{A}\right)$. Then $A$ and $B$ would be $\mathbb{Q} H H$ 's embedded in a $\mathbb{Z} H S$. It follows from Lemma 6.1 that $A$ and $B$ would be $\mathbb{Z H H}$ 's. Thus, by Lemma 2.2, the surgery would preserve the integral Alexander module. 


\section{References}

[AL05] E. AuClair \& C. LeSCOP - "Clover calculus for homology 3-spheres via basic algebraic topology", Algebr. Geom. Topol. 5 (2005), p. 71-106 (electronic).

[Bla57] R. C. BLANChFIELD - "Intersection theory of manifolds with operators with applications to knot theory", Ann. of Math. (2) 65 (1957), p. 340-356.

[FM12] B. Farb \& D. Margalit - A primer on mapping class groups, Princeton Mathematical Series, vol. 49, Princeton University Press, Princeton, NJ, 2012.

[GGP01] S. Garoufalidis, M. Goussarov \& M. PolyaK - "Calculus of clovers and finite type invariants of 3-manifolds", Geom. Topol. 5 (2001), p. 75-108 (electronic).

[GK04] S. Garoufalidis \& A. KRICKER - "A rational noncommutative invariant of boundary links", Geom. Topol. 8 (2004), p. 115-204 (electronic).

[GR04] S. Garoufalidis \& L. RozAnsky - "The loop expansion of the Kontsevich integral, the null-move and $S$-equivalence", Topology 43 (2004), no. 5, p. 11831210.

[Hab00] K. HABIRO - "Claspers and finite type invariants of links", Geom. Topol. 4 (2000), p. 1-83 (electronic).

[Kea75] C. Kearton - "Blanchfield duality and simple knots", Trans. Amer. Math. Soc. 202 (1975), p. 141-160.

[Lic97] W. B. R. LickORISH - An introduction to knot theory, Graduate Texts in Mathematics, vol. 175, Springer-Verlag, New York, 1997.

[Mat87] S. V. MATVEeV - "Generalized surgeries of three-dimensional manifolds and representations of homology spheres", Mat. Zametki 42 (1987), no. 2, p. 268278,345 .

[Mou12a] D. Moussard - "Finite type invariants of rational homology 3-spheres", arXiv:1203.1603v1, to appear in Algebraic and Geometric Topology, 2012.

[Mou12b] - , "On Alexander modules and Blanchfield forms of null-homologous knots in rational homology spheres", J. Knot Theory Ramifications 21 (2012), no. 5, p. 1250042,21 . 
[NS03] S. NAIK \& T. STANFORD - "A move on diagrams that generates $S$-equivalence of knots", J. Knot Theory Ramifications 12 (2003), no. 5, p. 717-724.

[Tro73] H. F. Trotter - "On S-equivalence of Seifert matrices", Invent. Math. 20 (1973), p. 173-207. 